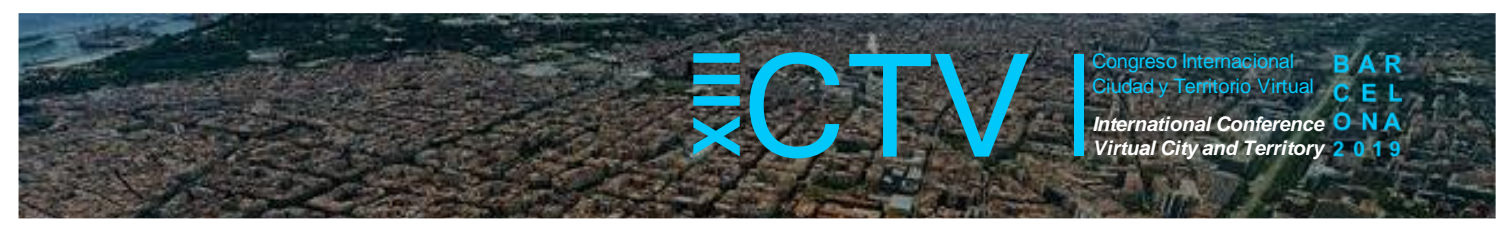

\title{
VARIACIONES ESTACIONALES DEL EFECTO DE ENFRIAMIENTO DE LOS PARQUES URBANOS DE BARCELONA: UNA APROXIMACIÓN MEDIANTE TELEDETECCIÓN
}

\author{
García-Haro, Alan ${ }^{1 *}$; Arellano, Blanca ${ }^{2}$; y Roca, Josep ${ }^{3}$ \\ Remisión inicial: dd-mm-2019; Aceptación definitiva: dd-mm-2019; Publicación: dd-mm-2019
}

Citación: García-Haro, A. et al. (2019). Variaciones estacionales del efecto de enfriamiento de los parques urbanos de Barcelona: una aproximación mediante teledetección. En XIII CTV 2019 Proceedings: XIII International Conference on Virtual City and Territory: "Challenges and paradigms of the contemporary city”: UPC, Barcelona, October 2-4, 2019. Barcelona: CPSV, 2019, p. 8957. E-ISSN 2604-6512. DOI http://dx.doi.org/10.5821/ctv.8957

\begin{abstract}
Resumen
Los parques urbanos representan espacios de oportunidad para la adaptación de las ciudades al cambio climático. Su comúnmente abundante vegetación y superficies permeables, rompen la continuidad de la cubierta de suelo artificial que distingue a las zonas urbanas, y generan una reducción de temperatura en relación a su contexto, que se extiende hacia sus alrededores y genera la llamada isla de frío de los parques urbanos ( $\mathrm{PCl}$ por sus siglas en inglés). Por lo tanto, reconocer el papel de las características físicas que definen este efecto, resulta una oportunidad para definir parámetros de adaptación climática de las ciudades y reducir riesgos a la salud durante episodios extremos de ola de calor. En años recientes, la inclusión de la Temperatura de la Superficie Terrestre (LST por sus siglas en inglés) a los estudios del comportamiento climático de los espacios urbanos, ha permitido abordar un mayor número de casos con información térmica simultánea en áreas de mayor escala. En este contexto, el presente trabajo plantea una aproximación mediante teledetección a la cuantificación de las variaciones estacionales del efecto de enfriamiento de los parques urbanos de Barcelona y la relación con su diseño y ubicación. Particularmente, se destaca la relación de los cambios anuales en la composición de los espacios verdes al interior y alrededores de los parques, con su comportamiento microclimático. Para esto, primero se calcula la extensión $\left(L_{\max }\right)$ e intensidad $\left(\Delta T_{\max }\right)$ del efecto de enfriamiento de 86 parques urbanos de la ciudad de Barcelona a partir de un método de homogenización de la temperatura de los alrededores mediante anillos concéntricos, basado en la $L S T$ del satélite Landsat-8 OLI/TIRS de días representativos de las cuatro estaciones del año. Posteriormente, se analiza la correlación entre dichos indicadores y los atributos físicos de forma, ubicación y las variaciones anuales en la composición de los espacios verdes de los parques y sus alrededores. Las últimas, obtenidas mediante la cuantificación de los cambios del Índice de Vegetación de Diferencia Normalizada (NDVI por sus siglas en inglés) obtenido de las mismas imágenes satelitales.
\end{abstract}

Los primeros resultados registraron que el conjunto de parques urbanos de Barcelona presenta una LST media por estación entre 0.99 y $2.56^{\circ} \mathrm{C}$ inferior que el contexto urbano. Donde se registraron valores medios de $\mathrm{PCl}$ por estación de 57.91 a $78.02 \mathrm{~m}$ de $L_{\max }$ y de 0.75 a $1.70^{\circ} \mathrm{C}$ de $\Delta T_{\max }$. Además, durante todo el año, 83 parques registraron $P C /$ positiva y uno registró negativo todo el año. El mayor número de parques registraron su máxima $P C l$ durante verano y su mínimo en invierno. Por otra parte, el conjunto de parques registra un NDVI medio entre 0.17 a 0.22 mayor que el contexto urbano de Barcelona. Donde, el rango de variación anual de NDVI de cada parque no registró correlación con las variaciones de su efecto de enfriamiento. Asimismo, en coincidencia con la literatura, la $\Delta T_{\max }$ resultó como el indicador de la $\mathrm{PCl}$ con mayor correlación con los atributos físicos durante todo el año. Particularmente, destaca que cuanto mayor sea la proporción de áreas con árboles agrupados identificados con un NDVI entre 0.50 a 0.60 , mayor es la $\Delta T_{\max }$ en las cuatro estaciones, mientras que el resto de atributos varía en cada estación. En cuanto a la $L_{\max }$ no registró una correlación significativa constante durante el año con algún atributo físico, aunque si con la $\Delta T_{\max }$. En general, los resultados apuntan a una predominante influencia de las características físicas de la forma de parque y la composición de sus espacios verdes. Dejando de lado su ubicación y las características de su contexto urbano.

Ante esto, en las conclusiones se discute la pertinencia de la aproximación metodológica al caso estudiado y las aportaciones al reconocimiento de la influencia del diseño y ubicación de los parques urbanos de Barcelona sobre su influencia microclimática. El presente estudio se desarrolla en el marco del proyecto "Urban-CLIMPLAN. La isla de calor

\footnotetext{
1 Universidad Politécnica de Cataluña, Departamento de Tecnología de la Arquitectura, Centro de Política de Suelo y Valoraciones http://orcid.org/0000-0001-7128-3667; ${ }^{2}$ https://orcid.org/0000-0002-4302-6492; ${ }^{3}$ https://orcid.org/00000003-3970-65053. * Correo de contacto: alan.garcia.haro@upc.edu
} 


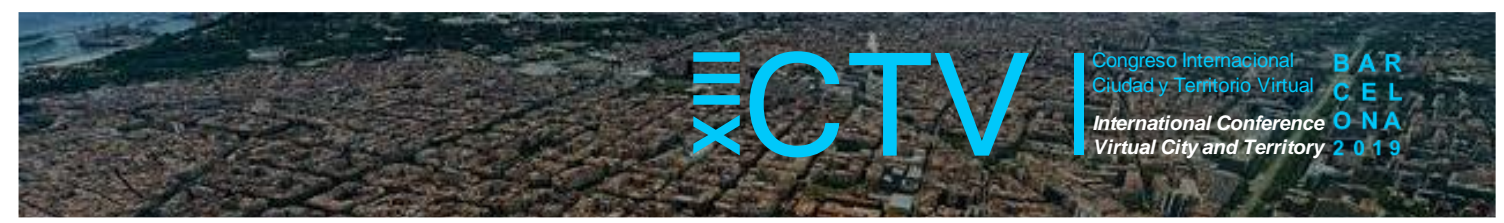

urbana: efectos en el cambio climático y modelado para estrategias de planeamiento territorial y urbano. Aplicación a la Región Metropolitana de Barcelona"; financiado por el Ministerio de Economía y Competitividad de España (MINECO) y el Fondo Europeo para el Desarrollo Regional (FEDR).

\section{Abstract}

Urban parks are spaces of opportunity for the adaptation of cities to climate change. Its commonly abundant vegetation and unsealed surfaces, break the continuity of the artificial land cover that distinguishes urban areas, and generate a temperature reduction in relation to its context, which extends towards its surroundings and generates the so-called urban park cool island $(\mathrm{PCl})$. Therefore, recognizing the role of the physical characteristics that define this effect is an opportunity to propose parameters of climate adaptation of cities and reduce health risks during extreme heat wave episodes. In recent years, the addition of the Land Surface Temperature (LST) to studies of the climatic behavior of urban spaces, has allowed addressing a greater number of cases with simultaneous thermal information in larger-scale areas. In this context, in this work we propose a remote sensing approach to the quantification of the seasonal variations of the cooling effect of the urban parks of Barcelona and the role of their design and location. Particularly, we highlight the relationship of the annual changes in the composition of the green spaces inside and around the parks with their microclimatic behavior. For this purpose, we calculate the $\mathrm{PCl}$ limit extension $\left(L_{\max }\right)$ and intensity $\left(\Delta T_{\max }\right)$ of 86 urban parks in the city of Barcelona, through a method of homogenization of the surrounding temperature using concentric rings, based on the LST of Landsat$8 \mathrm{OLI} / \mathrm{TIRS}$ satellite of representative days of the four seasons of the year. Subsequently, we analyze the correlation between these indicators and the physical attributes of shape, location and annual variations in the composition of the green spaces of the parks and their surroundings. Where we obtained the latter by quantifying changes in the Normalized Difference Vegetation Index (NDVI) from the same satellite images.

First results record that the urban parks in Barcelona has an average LST per season between 0.99 and $2.56^{\circ} \mathrm{C}$ lower than the urban context. Where we register average PCl values of 57.91 to $78.02 \mathrm{~m}$ of $L_{\max }$ and 0.75 to $1.70^{\circ} \mathrm{C}$ of $\Delta T_{\max }$. In addition, throughout the year, 83 parks register positive $\mathrm{PCl}$ and only one negative the entire year. Most of the parks record their maximum $\mathrm{PCl}$ during summer and their minimum in winter. On the other hand, the set of parks records an average NDVI of 0.17 to 0.22 greater than the urban context of Barcelona. Where, the annual variation range of NDVI of each park does not correlate with its $\mathrm{PCl}$ variations. In addition, coinciding with the literature, $\Delta T_{\max }$ is the indicator of the $\mathrm{PCl}$ with the highest correlation with physical attributes throughout the year. Particularly, the greater the proportion of areas with clustered trees (identified with an NDVI from 0.50 to 0.60 ), the greater the $\Delta T_{\max }$ in the four seasons, while the rest of the attributes vary in each season. As for the $L_{\max }$, it does not register a constant significant correlation during the year with some physical attribute, although with the $\Delta T_{\max }$ does. In general, the results point to a predominant influence of the physical characteristics of the park shape and the composition of its green spaces. Leaving aside its location and the characteristics of its urban context.

Given this, as conclusions we discuss the relevance of the methodological approach to the case studied and the contributions to the recognition of the influence of the design and location of urban parks in Barcelona on their microclimatic influence. The present study is part of the project "Urban-CLIMPLAN. The urban heat island: effects on climate change and modeling for territorial and urban planning strategies. Application to the Metropolitan Region of Barcelona"; financed by the Ministry of Economy and Competitiveness of Spain (MINECO) and the European Fund for Regional Development (FEDR).

Palabras Clave: isla de frío de los espacios verdes; isla de calor urbana; microclima urbano

Key words: green spaces cool island; urban heat island; urban microclimate

\section{Introducción}

Los parques urbanos son fundamentales en la mitigación del calentamiento de las ciudades en cara al cambio climático. Comúnmente con predominante vegetación y superficies permeables, los parques presentan una reducción de temperatura en relación a su entorno urbano, la cual se suele extender hacia sus alrededores cercanos, generando un efecto de "isla de frío" ( $\mathrm{PCl}$ por sus siglas en inglés) (Spronken-Smith \& Oke, 1998). Dicho comportamiento se debe a la ruptura de la continuidad de las características que propician la creación de la isla de calor urbana (UHI por sus siglas en inglés) (Oke, 1982). 


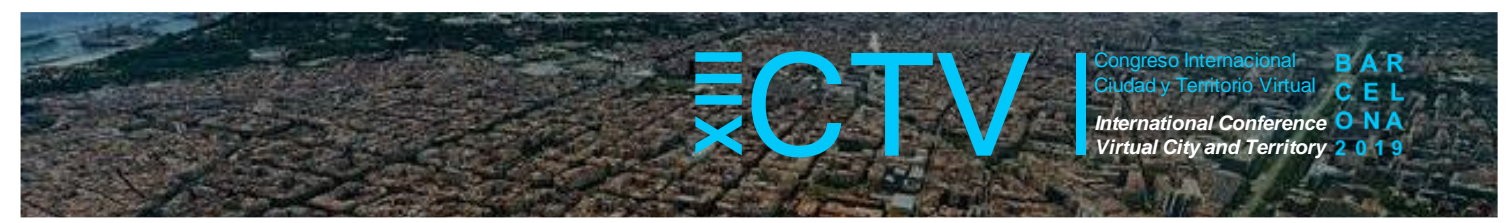

Desde la perspectiva de la planeación y diseño urbano, los parques son espacios versátiles y potenciales puntos de atención de las problemáticas urbanas relacionadas al cambio climático. Debido a sus características, estos espacios tienen más flexibilidad de transformación que su entorno urbano edificado. Dicha cualidad, ha impulsado el desarrollo de estudios de evaluación y reconocimiento de la influencia microclimática de los elementos físicos que los componen. En términos de características físicas, la literatura reconoce ampliamente la calidad de la vegetación como uno de los atributos más influyentes en la caída de temperaturas de los espacios urbanos (Bowler, Buyung-Ali, Knight, \& Pullin, 2010). No obstante, los estudios de casos específicos revelan otros factores que definen el efecto de enfriamiento de los parques, tales como los patrones de forma de los elementos del paisaje en su interior (Cao, Onishi, Chen, \& Imura, 2010) y las características de su entorno. Donde destaca la morfología del contexto urbano, condiciones geográficas (Hamada, Tanaka, \& Ohta, 2013), régimen meteorológico y variaciones estacionales. Donde los resultados apuntan a una mayor manifestación del efecto de enfriamiento de los parques inmersos dentro de un entorno de mayores temperaturas (Hamada \& Ohta, 2010).

En este contexto, aquí se plantea una aproximación a la cuantificación del efecto de enfriamiento de los parques urbanos de Barcelona y sus variaciones estacionales mediante teledetección. Donde se busca hacer una identificación generalizada de la influencia de las características físicas dentro y alrededor de los parques, sobre su efecto de enfriamiento. Particularmente, el estudio aquí desarrollado se propone como parte de una serie de estudios de la influencia climática de los espacios verdes dentro de la Región Metropolitana de Barcelona. En los cuales se ha registrado la influencia de la distribución de los tipos de superficie y vegetación sobre las temperaturas en casos específicos, mediante el análisis de mediciones de campo de temperatura de aire $\left(T_{A}\right)$ y superficie $\left(T_{S}\right)$. Donde, durante verano, los jardines de Coll Favà con predominante superficie pavimentada y una baja proporción de vegetación, registraron una $\mathrm{T}_{\mathrm{A}} \mathrm{y} \mathrm{T}_{\mathrm{S}}$ diurna 5.03 y $9.911^{\circ} \mathrm{C}$ respectivamente, más elevada que la del Parc Central mayormente cubierto por vegetación densa, ambos en el municipio de Sant Cugat del Vallès (Roca, Arellano, \& Batlle, 2018). Posteriormente, otro estudio registró que el Parc Turó y el Parc del Centre del Poblenou registraron 80 y $90 \mathrm{~m}$ de extensión de su $P C l$ sobre sus alrededores durante el día, y una reducción de temperatura de 2.89 y $2.75^{\circ} \mathrm{C}$ respectivamente. Donde se identificó que la vegetación al interior del parque tiene la mayor influencia sobre la reducción de temperatura en el parque, mientras que la extensión de la $\mathrm{PCl}$ se ve fuertemente influenciado por la configuración del entorno urbano. Asimismo, en un estudio mediante métodos de análisis espacial con datos de teledetección en la conurbación de Viladecans, Gavà y Castelldefels, se buscó hacer una cuantificación generalizada del efecto de enfriamiento de los espacios verdes en la escala local y su relación con los atributos físicos del territorio. Identificando que cada $100 \mathrm{~m}$ de alejamiento desde los espacios verdes, la temperatura de la superficie terrestre (LST por sus siglas en inglés) de las áreas urbanas tiende a incrementar por $1.81^{\circ} \mathrm{C}$, y donde siete parques como casos de estudio registraron un efecto de enfriamiento que alcanza una extensión media de $93.33 \mathrm{~m}$ sobre su entorno urbano con una reducción de temperatura de $1.22^{\circ} \mathrm{C}$ (Arellano, García-Haro, \& Roca, 2019).

La ciudad de Barcelona es de especial interés debido a su composición urbana de alta densidad y relevancia en el litoral del Mar Mediterráneo. Con apenas un territorio de $10 \mathrm{~km}$ de largo, Barcelona alberga a 1.62 millones de habitantes y representa la cabecera de la Región Metropolitana de Barcelona, una de las principales zonas metropolitanas de España (Statistical Office of the European Union, 2018). 


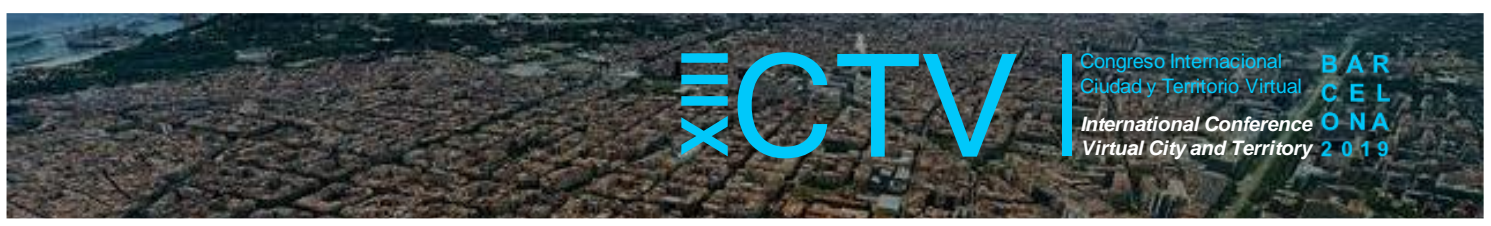

\section{Datos y métodos}

\subsection{Casos de estudio}

Se seleccionaron 86 parques urbanos dentro del municipio de Barcelona, España (Figura 1)

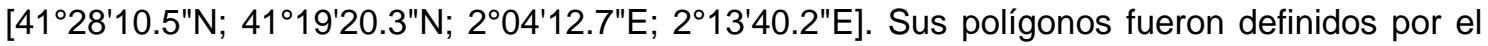
Área de Ecología y Servicios Urbanos del Ayuntamiento de Barcelona (Area d'Ecologia i Serveis Urbans de Barcelona, 2017) y algunos son jardines o plazas con instalaciones deportivas y culturales, sin predominante presencia de vegetación o superficies permeables.

\section{Figura 1. Ubicación de los parques urbanos y estaciones meteorológicas en Barcelona}

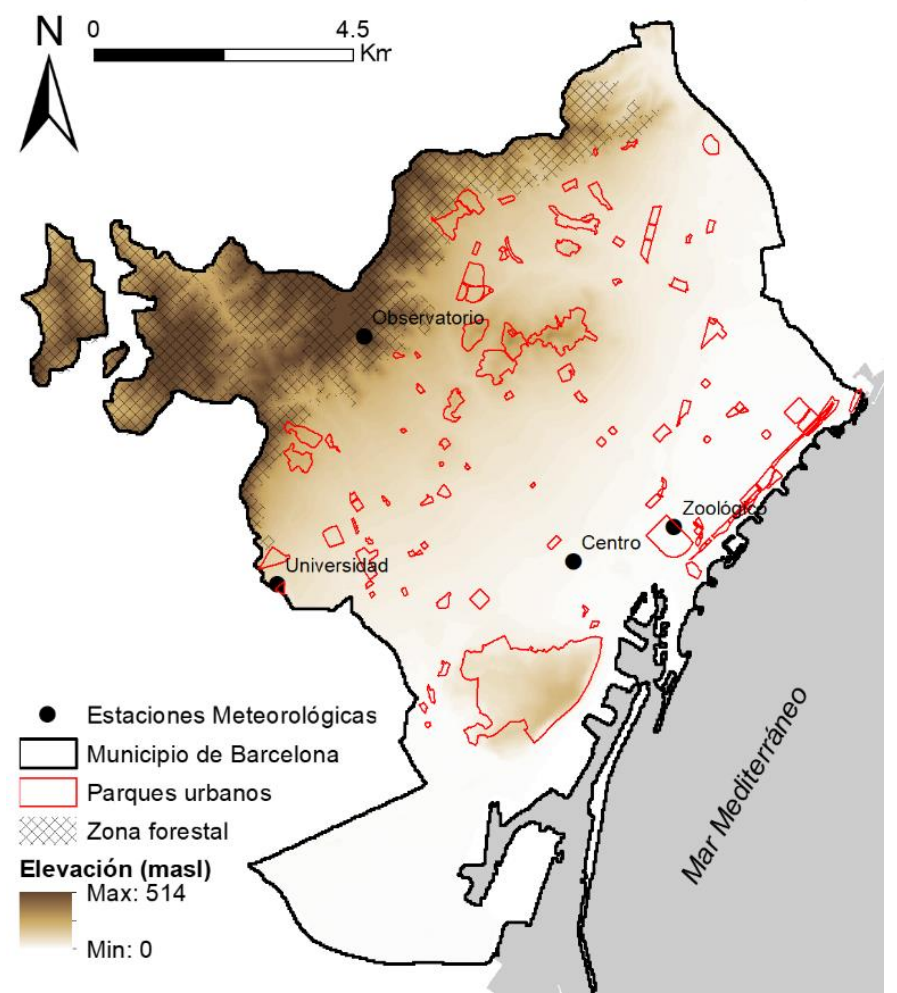

Fuente: Elaboración con información del Area d'Ecologia i Serveis Urbans de Barcelona (2017), Servei Meteorològic de Catalunya (2018), Departament de Territori i Sostenibilitat (2016) e Institut Cartogràfic i Geològic de Catalunya (2017).

\subsection{Obtención de registro de temperaturas}

Se recuperó la $L S T$ de Landsat-8 OLI/TIRS de los días 31 de enero, 21 de abril, 17 de julio y 23 de octubre de 2018, representativos de invierno, primavera, verano y otoño respectivamente. Se calculó mediante el método de corrección de emisividad (Stathopoulou \& Cartalis, 2007) como:

$$
L S T_{C}=T_{B}\left[1+\left(\lambda \times T_{B} / \alpha\right) \ln \varepsilon\right]
$$

Donde $L S T_{C}$ es la $L S T$ en ${ }^{\circ} \mathrm{C}, T_{B}$ es la temperatura de brillo del sensor calculada con la radiancia de la banda térmica infrarroja uno (TIRS1). Mientras que $\lambda$ es el valor central del ancho de onda de la banda TIRS1 $(10.895 \mu \mathrm{m})$ y $\alpha$ es la constante de la radiación superficial $(1.4388 \times 10-2 \mathrm{mK})$. La $\varepsilon$ es la emisividad espectral de la superficie, estimada mediante la limitación de NDVI (Sobrino, 


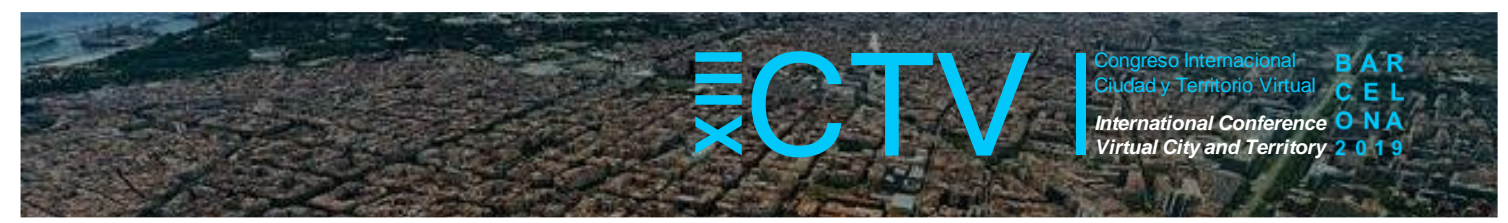

Jiménez-Muñoz, Soria, Romaguera, \& Guanter, 2008) con valores de emisividad de 0.95 para suelo desnudo debajo del $N D V I_{\min }(0.2), 0.98$ para superficies con predominante cubierta de vegetación arriba de $N D V I_{\max }(0.5)$ y $0.017 P v+0.963$ para áreas de cubierta mixta (Stathopoulou, Cartalis, \& Petrakis, 2007). Pv es la proporción de vegetación de las celdas mixtas y se calcula como [(NDVI - NDVImin) / (NDVI max $\left.\left.-N D V I_{\min }\right)\right]^{2}$. Mientras que el $N D V I$ se calculó con las bandas roja e infrarroja cercana $(N I R)$ como $[(\rho N I R-\rho R E D) /(\rho N I R+\rho R E D)]$, donde $\rho$ se refiere a la reflectancia de la banda. Los valores de $N D V I$ que van de -1 a +1 , de menos a más vegetación. Así como ambos LST y NDVI tienen una resolución de $30 \mathrm{~m}$ celda.

Asimismo, se obtuvo la temperatura de aire $\left(T_{A}\right)$ correspondiente a los días y hora del registro de la imagen satelital. Donde se recurrió a las cuatro estaciones meteorológicas disponibles dentro del municipio (Figura 1), Centro, Observatorio, Universidad y Zoológico.

\subsection{Cuantificación del efecto de enfriamiento}

Se calculó la extensión e intensidad de la isla de frío de cada parque $(P C /$ por sus siglas en inglés) a partir de una serie ajustada de valores de $L S T$ de los alrededores ordenados por distancia al parque. Donde la extensión se define como el límite máximo de la curva de enfriamiento $\left(L_{\max }\right)$; y la intensidad se calcula como la diferencia de temperatura entre $L_{\max }$ y el parque $\left(\Delta T_{\max }\right)$ (Cao, Onishi, Chen, \& Imura, 2010).

Para generar las series de $L S T$, se propuso un radio de $500 \mathrm{~m}$ alrededor de cada parque como área de potencial influencia (Roca, Arellano, \& Batlle, 2018). Se dividió en 50 anillos concéntricos de $10 \mathrm{~m}$ de ancho y se calculó la $L S T$ media de cada uno $\left(T_{R}\right)$ y la correspondiente al parque $\left(T_{P}\right)$, excluyendo los valores de celdas dentro de otros parques, el mar y la zona forestal (Figura 1). Obteniendo 86 series de datos con 51 valores de $L S T$, la de los parques y sus 50 anillos concéntricos a su respectiva distancia $\left(L_{R}\right)$. Finalmente, se ajustaron las fluctuaciones de las series mediante un ajuste de curva polinómico de ocho grados; que registró el mejor ajuste por medio de procesamiento en STATA.

Las series ajustadas de $L S T$ registraron tres tipos de comportamiento climático alrededor de los parques. Por lo que se propusieron métodos de definición de $L_{\max }$ en cada uno:

1. $P C /$ nula. $L_{\max }$ igual a cero cuando la $L S T$ media fue mayor que los primeros tres anillos concéntricos $(30 \mathrm{~m})$, previendo la influencia del tamaño de la celda de la banda.

2. Curva de enfriamiento y descenso de temperatura. Se definió el primer punto de cambio de tendencia de positivo a negativo como punto de $L_{\max }$.

3. Curva de enfriamiento y aumento de temperatura. Se definió $L_{\max }$ como el primer punto con cambio de gradiente nulo. Donde, se calculó el gradiente de LST entre cada par de anillos $\left[m_{R}=\left(T_{R+1}-T_{R}\right) /\left(L_{R+1}-L_{R}\right)\right]$, se computó la diferencia entre cada par de gradientes contiguos [ $\Delta m_{R}=m_{R+1}-m_{R}$ ] y se estimó la diferencia entre la máxima y mínima diferencia de gradiente $\left(\Delta m_{R}\right)$ de la serie $i\left[{ }^{r a n g o} \Delta m_{R, i}={ }^{\max } \Delta m_{R, i}{ }^{m i n} \Delta m_{R, j}\right]$. Así, el primer anillo donde $\Delta m_{R}$ esté dentro del $\pm 1 \%$ de rango $\Delta m_{R, i}$ es el punto de $L_{\max }$.

Entonces, el menor de los tres valores se definió como $L_{\max }$. De la cual se calculó la $\Delta T_{\max }$ como $T_{U}-T_{P}$ (Spronken-Smith \& Oke, 1998), donde $T_{U}$ es la temperatura urbana y corresponde a la $L S T$ media del anillo $L_{\max }$. Los resultados de $L_{\max }$ y $\Delta T_{\max }$ se expresan en metros y ${ }^{\circ} \mathrm{C}$. 


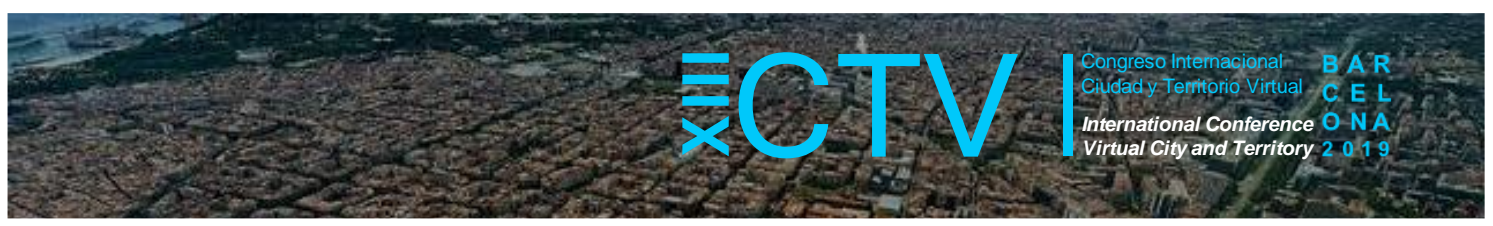

\subsection{Obtención de descriptores físicos}

Se estimaron los descriptores físicos de los parques y sus alrededores a partir de tres subdivisiones espaciales: 1) el parque; 2 ) la superficie hasta el $L_{\max }$ (Área $L_{\max }$ ); y 3 ) aquella entre la $L_{\max }$ y el límite del radio de $500 \mathrm{~m}$ (Fuera $L_{\max }$ ). Donde, debido a las variaciones anuales de $L_{\max }$, dichas subdivisiones cambian entre cada temporada.

Por otra parte, los descriptores físicos se clasificaron en tres tipos: 1) ubicación; 2) forma de los parques; y 3) composición de los espacios verdes al interior y alrededor del parque. Los atributos de ubicación son la elevación (metros sobre el nivel del mar; masl), distancia al mar y distancia a la zona forestal. Mientras que también se consideró como atributo geográfico la pendiente del terreno (\%). Por su parte, la forma del parque se describe por su tamaño (hectáreas; ha) e Índice de Forma del Paisaje ( $L S I$, por sus siglas en inglés). Este último calculado como la relación entre el área $(A)$ y el perímetro $(P)$ del parque $[L S I=P / 2(\sqrt{ } \pi \times A)]$, donde un mayor $L S I$ indica mayor complejidad (McGarigal \& Marks, 1995)

Los espacios verdes se caracterizaron por porcentaje de vegetación dentro de rangos de NDVI con diferentes niveles de vegetación (Figura 2). Se clasificaron las superficies por rangos de 0.10 $\mathrm{NDVI}$ entre 0.20 y $0.60 \mathrm{NDVI}$, correspondientes a los límites del suelo con cubierta mixta, donde $N D V I<0.20$ son las áreas sin vegetación, con suelo permeable, pavimentado o construido; y $N D V 1>0.60$ contiene áreas predominantemente cubiertas por vegetación.

Figura 2. Proporción y tipo de vegetación por de rangos de NDVI en celdas del satélite

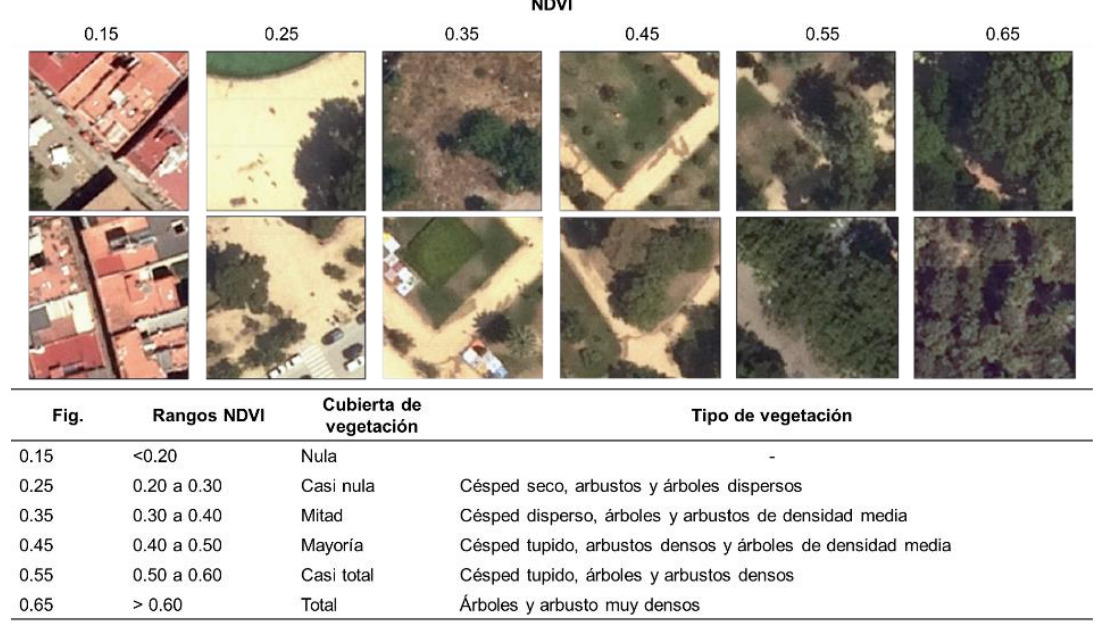

Fuente: Elaboración propia con información de imagen de $0.25 \mathrm{~m}$ de resolución tomada del Institut Cartogràfic i Geològic de Catalunya (2017).

\subsection{Cálculo de correlaciones}

Primero, se calculó el valor de correlación bivariada (Pearson) de los todos los descriptores físicos con los indicadores de $\mathrm{PCl}$. Para posteriormente identificar los descriptores físicos explicativos de cada uno de los indicadores de enfriamiento mediante una regresión lineal múltiple paso por paso. Donde, se discriminaron las variables identificadas con multicolinealidad y aquellas no significativas hasta alcanzar el modelo con el mayor $R^{2}$. En ambos cálculos se 


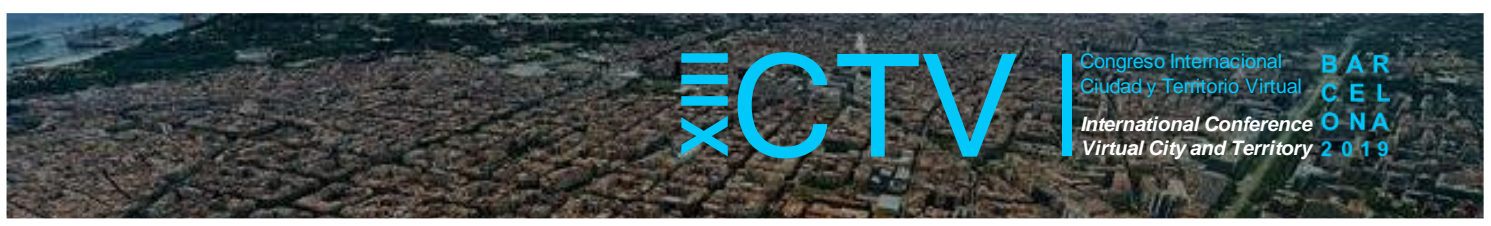

integraron los 86 parques, aunque en la correlación con los atributos de los alrededores urbanos, únicamente se contemplaron los casos de $\mathrm{PCl}$ positiva.

En general, se estimó la correlación para las cuatro estaciones. Donde la única variación física anual es el $N D V I$ y el porcentaje de superficie entre sus diferentes rangos.

\section{Resultados}

\subsection{Comportamiento climático de la ciudad de Barcelona}

Primeramente, la $T_{A}$ en las cuatro estaciones meteorológicas dentro del municipio de Barcelona, registró un comportamiento anual inconstante en el territorio (Figura 3). Donde la mayor diferencia entre estaciones meteorológicas se registró en invierno y otoño. En otoño se observa una diferencia $7.20^{\circ} \mathrm{C}$ entre el Zoológico y el Observatorio como máximo y mínimo respectivamente, en invierno de $4.40^{\circ} \mathrm{C}$ entre el Centro y el Zoológico, en primavera $2.30^{\circ} \mathrm{C}$ entre Universidad y el Zoológico, mientras que en verano se registró una diferencia de también $2.30^{\circ} \mathrm{C}$, pero entre la Zona Universitaria y el Observatorio. Desde otra perspectiva, la variación anual más amplia en una misma estación se registró en el Zoológico con $19.00^{\circ} \mathrm{C}$ entre verano e invierno. Seguida de Universidad con $17.60^{\circ} \mathrm{C}$, el Observatorio con $15.40^{\circ} \mathrm{C}$ y por último el Centro con una variación de $14.70^{\circ} \mathrm{C}$. Dichos valores, reflejan variaciones anuales irregulares en relación al territorio municipal y las características particulares de algunos espacios. En invierno, la $T_{A}$ más elevada se registra en el Centro de la ciudad y la más baja en el Zoológico; en primavera, la más alta se registra en Universidad y la más baja de nuevo en el Zoológico; en verano de nuevo Universidad registra la $T_{A}$ más elevada con el Observatorio como la más baja; y en otoño, se registró la $T_{A}$ más elevada en el Zoológico y la más baja en el Observatorio. De esta manera, aunque el Centro de la ciudad registra el segundo valor más elevado el resto del año el efecto de $U H I$ de Barcelona se ve reflejado con mayor claridad en el periodo invernal.

\section{Figura 3. $T_{A}\left({ }^{\circ} \mathrm{C}\right)$ en estaciones meteorológicas de Barcelona}

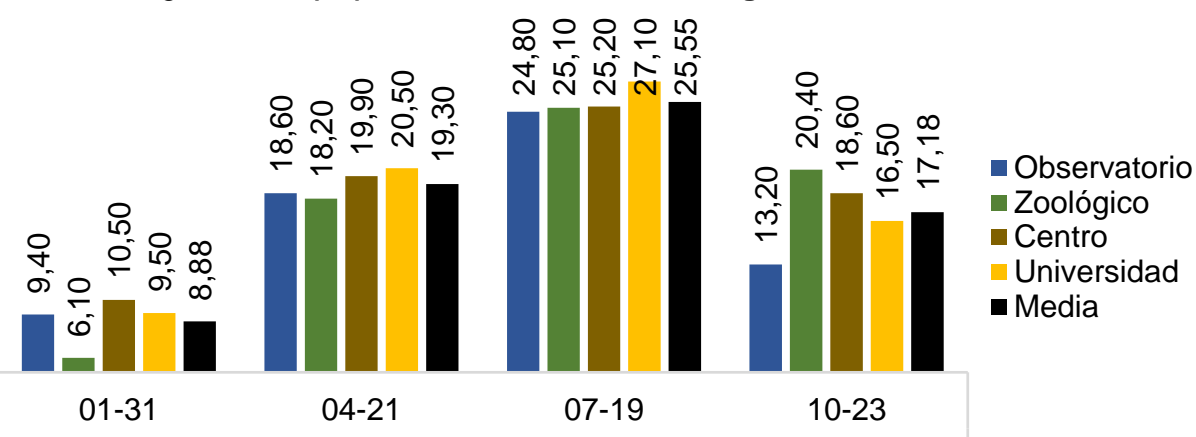

Fuente: Elaboración propia con información del Servei Meteorològic de Catalunya (2018)

Por otra parte, la relación entre la $L S T$ y el $N D V I$ de las superficies de los parques, bosques y el resto de espacios urbanos, indicó un comportamiento constante en los cuatro periodos del año (Figura 4). Donde los espacios urbanos registraron la LST más elevada, seguidos de los parques urbanos y por último los bosques (Tabla 1). En cuanto al NDVI, son los bosques los que registran los valores más altos en las cuatro estaciones, seguidos por los parques y con los espacios urbanos al final. Este mismo comportamiento se refleja en la amplitud de la variación anual de temperatura. Con la mayor diferencia de temperatura entre el periodo cálido y frío con $21.26^{\circ} \mathrm{C}$, 


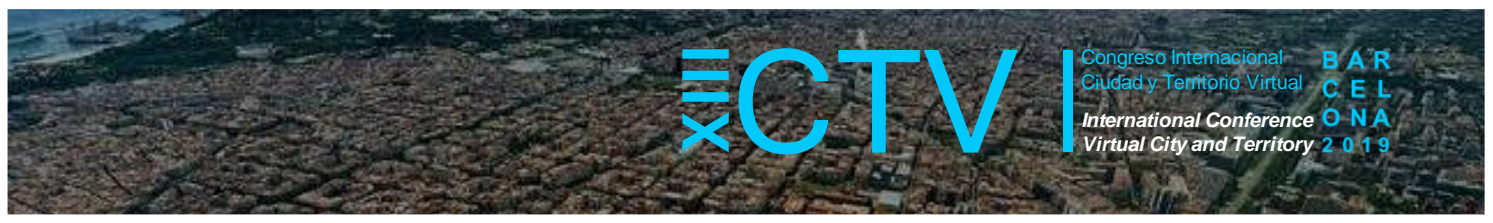

seguido de los parques urbanos con $19.84^{\circ} \mathrm{C}$ y al final los bosques con $17.42^{\circ} \mathrm{C}$. Dichas diferencias reflejan la inercia térmica de las zonas con mayor cantidad de vegetación, con una mayor resistencia al alza de temperaturas durante el periodo estío.

Figura 4. LST y NDVI de las cuatro temporadas del año
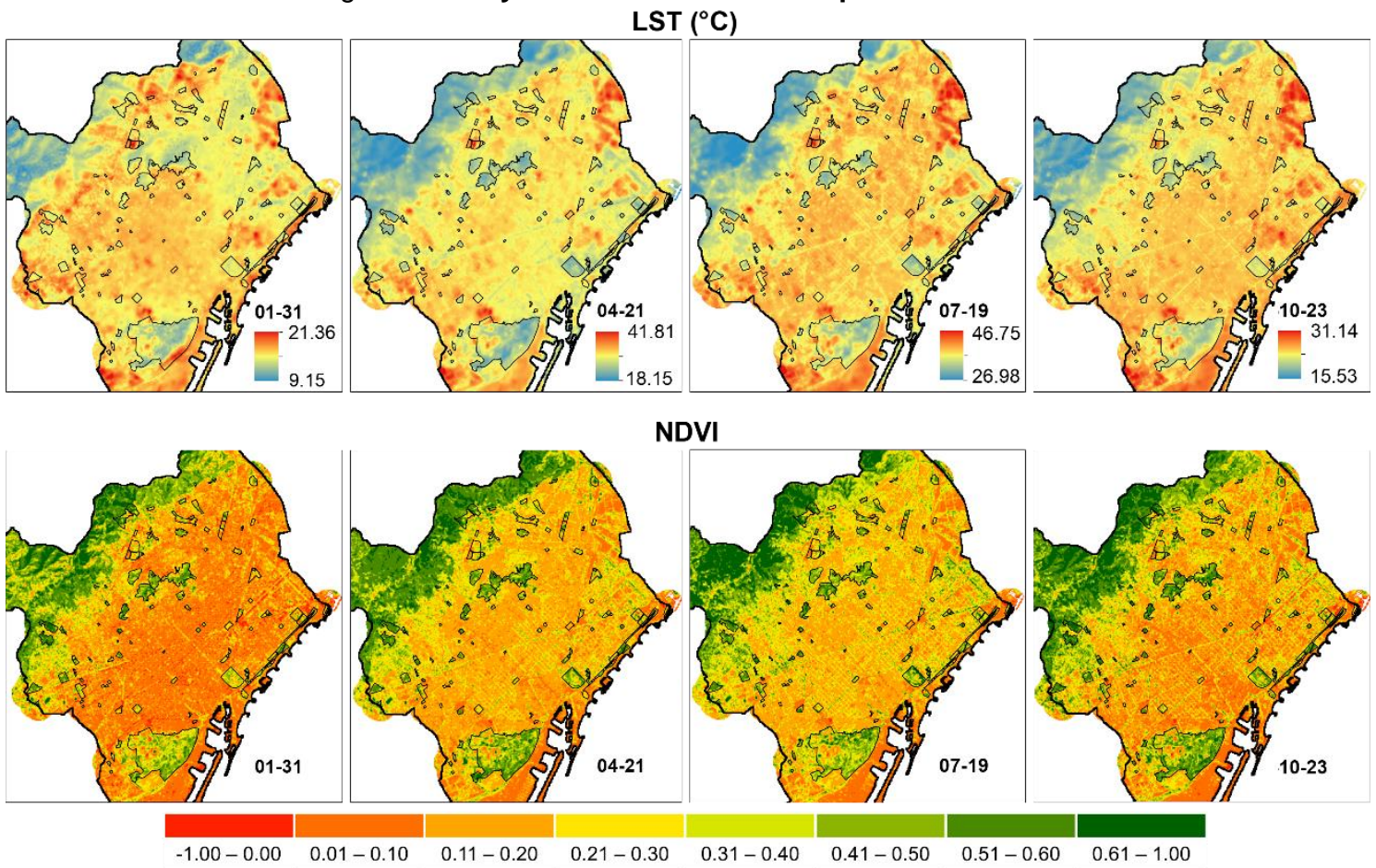

Fuente: Elaboración propia con información del U.S. Geological Survey (2018)

Desde una primera aproximación al efecto de enfriamiento de los parques, la superficie total de éstos, registra menor $L S T$ que los espacios urbanos durante todo el año (Tabla 1). Con una diferencia máxima de $2.56^{\circ} \mathrm{C}$ en primavera, seguida de $2.42^{\circ} \mathrm{C}$ en verano, $1.80^{\circ} \mathrm{C}$ en otoño y $0.99^{\circ} \mathrm{C}$ en invierno. No obstante, los bosques registran una $L S T$ media que oscila entre los $1.33^{\circ} \mathrm{C}$ y los $3.74^{\circ} \mathrm{C}$ menor que los parques en invierno y verano respectivamente. Dicha relación permite observar la inercia térmica o resistencia al calentamiento en la capa superficial que provee la predominante presencia de vegetación en los espacios, la cual no es una característica constante en todos los parques de Barcelona y se ve reflejado en que el mayor efecto de enfriamiento de éstos es en primavera cuando la $T_{A}$ media de las cuatro estaciones meteorológicas es $6.5^{\circ} \mathrm{C}$ menor que la de verano (Figura 3). Es decir, se puede esperar que las características físicas de algunos parques no garanticen resistencia al calentamiento de las superficies durante el verano.

Tabla 1. Diferencia de LST media en relación al conjunto de parques

\begin{tabular}{|l|c|c|c|c|c|c|c|c|}
\hline \multirow{2}{*}{ Espacio / Fecha } & \multicolumn{4}{c|}{ LST $\left({ }^{\circ} \mathbf{C}\right)$} & \multicolumn{3}{c|}{ LST (Diferencia Parque = 0) } \\
\cline { 2 - 10 } & $01-31$ & $04-21$ & $07-19$ & $10-23$ & $01-31$ & $04-21$ & $07-19$ & $10-23$ \\
\hline Municipio & 14.765 & 27.944 & 35.365 & 22.642 & 0.623 & 1.538 & 1.387 & 1.039 \\
\hline Bosque & 12.816 & 23.090 & 30.239 & 18.522 & -1.326 & -3.317 & -3.739 & -3.081 \\
\hline Urbano & 15.130 & 28.966 & 36.394 & 23.402 & 0.988 & 2.559 & 2.415 & 1.800 \\
\hline Parques & 14.142 & 26.407 & 33.979 & 21.603 & 0.000 & 0.000 & 0.000 & 0.000 \\
\hline
\end{tabular}

Fuente: Elaboración propia 


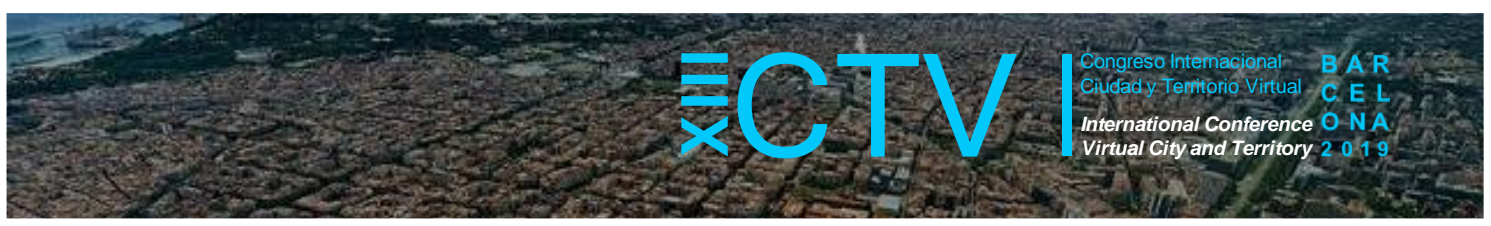

\subsection{Variaciones estacionales de la extensión del efecto de enfriamiento}

Se registró una extensión de enfriamiento media entre los $57.91 \mathrm{~m}$ en invierno y $78.02 \mathrm{~m}$ en verano (Tabla 2). Donde la máxima extensión de enfriamiento alcanzada por un parque fue en verano con $230 \mathrm{~m}$ y la mínima fue de 0 metros en las cuatro temporadas.

Tabla 2. Estadística descriptiva de $L_{\max }$ de las cuatro temporadas del año

\begin{tabular}{|l|c|c|c|c|}
\hline \multicolumn{1}{|l|}{$L_{\max }(\mathbf{m})$} & \multicolumn{1}{c}{$\mathbf{0 1 - 3 1}$} & $\mathbf{0 4 - 2 1}$ & $\mathbf{0 7 - 1 9}$ & $\mathbf{1 0 - 2 3}$ \\
\hline Max & 120.00 & 200.00 & 230.00 & 180.00 \\
\hline Min & 0.00 & 0.00 & 0.00 & 0.00 \\
\hline Mean & 57.91 & $\mathbf{7 2 . 4 4}$ & $\mathbf{7 8 . 0 2}$ & $\mathbf{7 1 . 2 8}$ \\
\hline Std. Dev. & 24.16 & 37.73 & 40.46 & 31.43 \\
\hline
\end{tabular}

Fuente: Elaboración propia

Tres parques registraron un $\mathrm{PCl}$ nula al menos durante tres temporadas del año: 1) el Parc de la Vall d'Hebron; 2) los Jardins de les Tres Xemeneies; y 3) el Parc de les Glòries. De los cuales, únicamente el Parc de la Vall d'Hebron resultó en nulo enfriamiento durante todo el año.

En primavera y verano los tres registraron efecto nulo, aunque en invierno Parc de les Glòries registró PCI positiva, así como los Jardins de les Tres Xemeneies en otoño. En dichos periodos, estos parques registraron una $L S T$ media superior que la de los $500 \mathrm{~m}$ alrededor, que alcanzó $2.39^{\circ} \mathrm{C}$ en primavera, lo que refleja un efecto de calentamiento del parque (Tabla 3 ).

Tabla 3. Variaciones estacionales de LST en parques y alrededores en fechas de PCI nulo

\begin{tabular}{|l|c|c|c|c|c|c|c|c|c|c|c|c|}
\hline LST $\left({ }^{\circ} \mathbf{C}\right)$ & \multicolumn{3}{|c|}{$\mathbf{0 1 - 3 1}$} & \multicolumn{3}{c|}{$\mathbf{0 4 - 2 1}$} & \multicolumn{3}{c|}{$\mathbf{0 7 - 1 9}$} & \multicolumn{3}{c|}{$10-23$} \\
\hline Parque & LST & $\begin{array}{c}\text { LST } \\
\mathbf{5 0 0 m}\end{array}$ & $\mathbf{\Delta T}$ & $\mathbf{L S T}$ & $\begin{array}{c}\text { LST } \\
\mathbf{5 0 0 m}\end{array}$ & $\mathbf{\Delta T}$ & $\mathbf{L S T}$ & $\begin{array}{c}\text { LST } \\
\mathbf{5 0 0 m}\end{array}$ & $\boldsymbol{\Delta T}$ & LST & $\begin{array}{c}\text { LST } \\
\mathbf{5 0 0 m}\end{array}$ & $\boldsymbol{\Delta T}$ \\
\hline $\begin{array}{l}\text { Parc de la } \\
\text { Vall } \\
\text { d'Hebron }\end{array}$ & 15.87 & 14.53 & -1.34 & 30.96 & 28.57 & -2.39 & 38.26 & 35.91 & -2.36 & 23.53 & 21.91 & -1.62 \\
\hline $\begin{array}{l}\text { Jardins de } \\
\text { les Tres } \\
\text { Xemeneies }\end{array}$ & 15.09 & 14.81 & -0.28 & 28.40 & 27.50 & -0.90 & 36.27 & 35.27 & -0.99 & - & - & - \\
\hline $\begin{array}{l}\text { Parc de les } \\
\text { Glóries }\end{array}$ & - & - & - & 30.59 & 28.83 & -1.77 & 37.85 & 36.59 & -1.26 & 25.24 & 23.85 & -1.40 \\
\hline
\end{tabular}

Fuente: Elaboración propia

En general, se observó que, en los periodos de mayor temperatura, la extensión de enfriamiento aumenta.

En invierno todos los parques registraron una extensión menor a $120 \mathrm{~m}$, con la mayoría concentrada entre los 40 y $70 \mathrm{~m}$ (Figura $5 \mathrm{a}$ ). Mientras que, en verano, algunos parques registraron un incremento importante en la extensión de enfriamiento (Figura 5c), con siete parques con más de $120 \mathrm{~m}$ de extensión y la mayoría concentrada entre los 50 y 100m. 


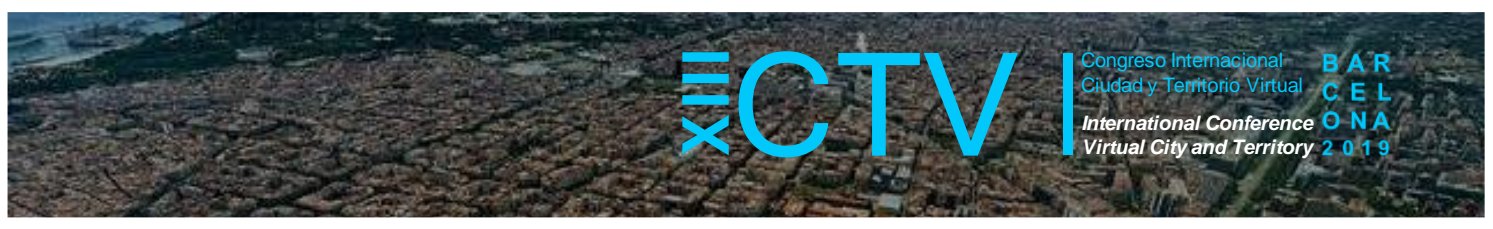

Figura 5. Histogramas de los parques por $L_{\max }$ las cuatro temporadas del año

a) Lmax 2018-01-31 18

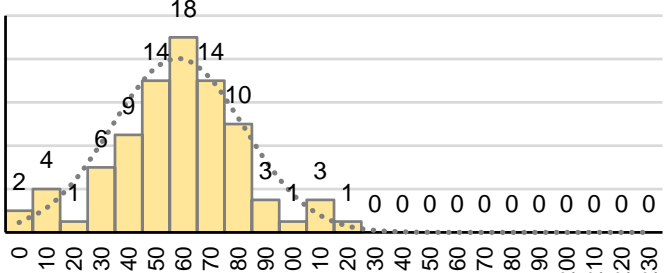

c) Lmax $2018-07-19$

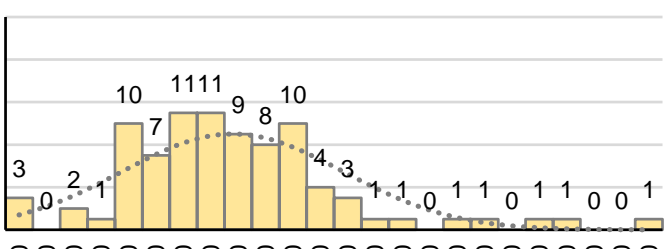

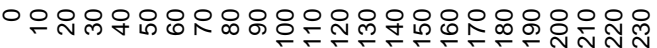

b) Lmax 2018-04-21

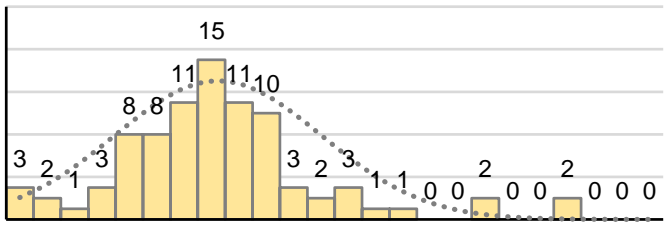

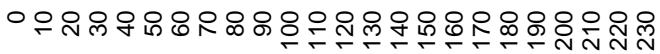

d) Lmax 2018-10-23

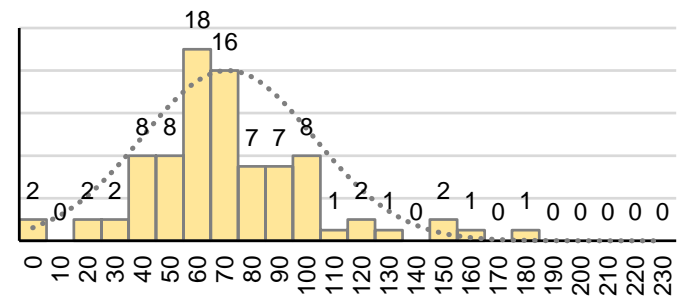

Fuente: Elaboración propia

De esta manera, se puede observar que, durante el invierno, el efecto de enfriamiento no se manifiesta claramente en los alrededores del parque. Por el contrario, en verano, aquellos parques con un alto potencial de enfriamiento, reflejan con mayor claridad la caída de temperatura en sus alrededores urbanos.

\subsection{Variaciones estacionales de la intensidad de enfriamiento}

Se registró una $\Delta T_{\max }$ media entre $0.75^{\circ} \mathrm{C}$ en invierno y $1.70^{\circ} \mathrm{C}$ en verano (Tabla 4). Al igual que la $L_{\max }$, la máxima $\Delta T_{\max }$ en un parque fue en verano con $3.67^{\circ} \mathrm{C}$ y la mínima fue de cero en las cuatro temporadas. De esta forma, se observa que en los periodos más cálidos la $\Delta T_{\max }$ aumenta.

En invierno todos los parques registraron una $\Delta T_{\max }$ inferior a los $2.00^{\circ} \mathrm{C}$, con la mayoría entre los 0.40 y $1.00^{\circ} \mathrm{C}$, mientras que, en verano, algunos parques registraron un aumento de $\Delta T_{\max }$ (Figura 6), con 38 parques arriba de $1.80^{\circ} \mathrm{C}$ de $\Delta T_{\max }$ y la mayoría entre 1.10 y $2.40^{\circ} \mathrm{C}$.

Tabla 4. Estadística descriptiva de $\Delta T_{\max }$ de las cuatro temporadas del año

\begin{tabular}{|c|c|c|c|c|}
\hline $\begin{array}{c}\Delta T \max \\
\left({ }^{\circ} \mathrm{C}\right)\end{array}$ & $01-31$ & 04-21 & $07-19$ & $10-23$ \\
\hline Max & 1.72 & 3.25 & 3.67 & 2.65 \\
\hline Min & 0.00 & 0.00 & 0.00 & 0.00 \\
\hline Mean & 0.75 & 1.51 & 1.70 & 1.31 \\
\hline Std. Dev. & 0.42 & 0.89 & 0.89 & 0.59 \\
\hline
\end{tabular}

Fuente: Elaboración propia 


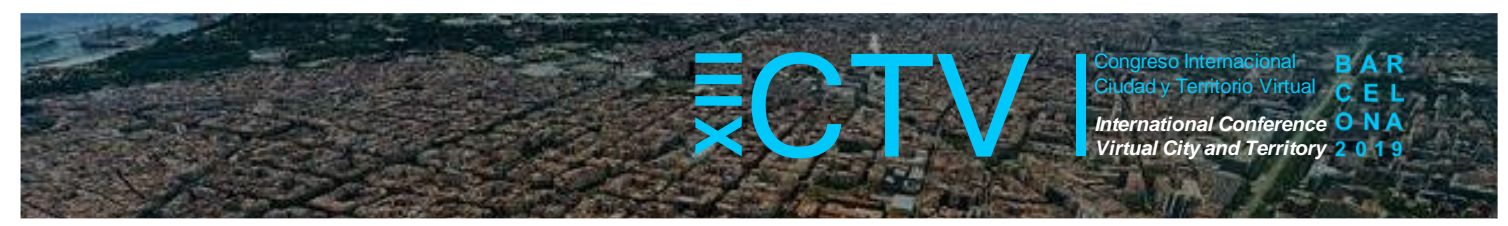

Figura 6. Histogramas de los parques por $\Delta T_{\max }$ las cuatro temporadas del año

a) $\Delta \operatorname{Tmax} 2018-01-31$

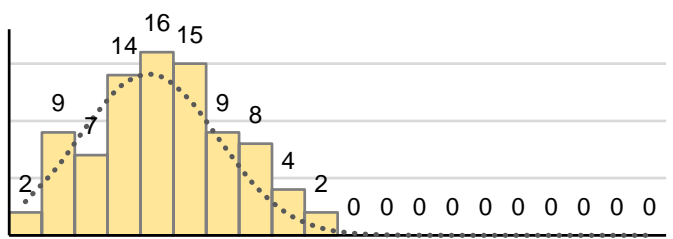

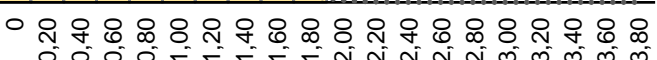

c) $\Delta \operatorname{Tmax} 2018-07-19$

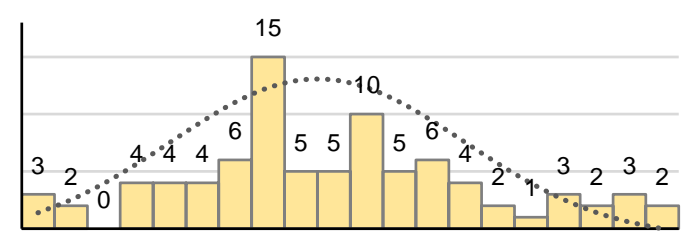

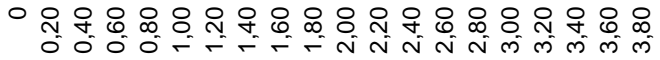

b) $\Delta \operatorname{Tmax} 2018-04-21$

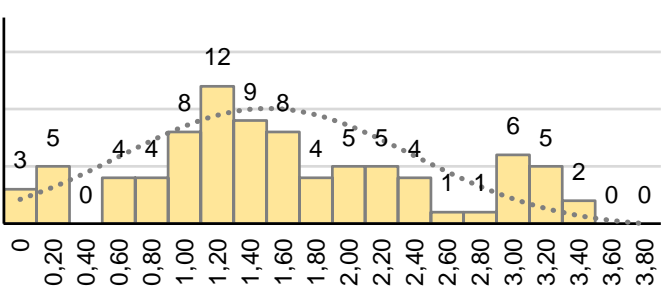

d) $\Delta \operatorname{Tmax} 2018-10-23$

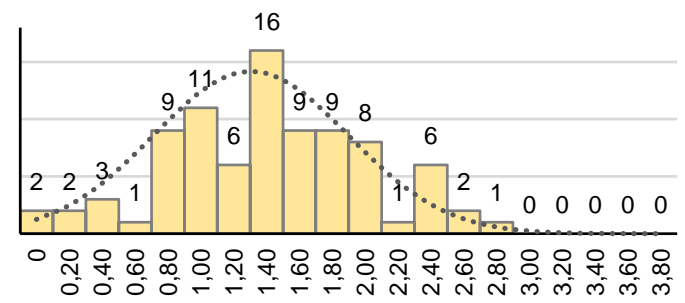

Fuente: Elaboración propia

Ante esto, se puede observar que la distribución de los parques en relación a su intensidad, se extiende hacia valores mayores, pero sin descartar los casos con intensidad inferior. Este comportamiento señala que, solamente algunos parques presentan características que mantienen el efecto de enfriamiento al interior de los parques durante los periodos de temperaturas elevadas. Mientras que los otros aumentan su temperatura en mayor proporción, reduciendo la diferencia de temperatura respecto a su entorno urbano.

\subsection{Descriptores físicos de forma y ubicación de los parques}

La forma y ubicación de los parques es constante durante todas las temporadas del año. Los 86 parques urbanos de Barcelona presentan una superficie media de $8.57 \mathrm{ha}$, oscilando entre 0.36 a 283.70ha. Particularmente, solo un parque presenta una superficie próxima a dicho máximo, mientras que el resto están por debajo de 39.38ha, con una superficie media de 5.33ha. En cuanto a su composición, los parques registraron un $L S I$ medio de 1.57 , que va desde 1.06 a 3.20. Dichas características dejan ver que los parques de Barcelona no corresponden a una forma semejante, correspondiendo a diferentes morfologías urbanas y equipamiento en su interior que atiende las necesidades funcionales de su contexto próximo.

Además, los parques se encuentran distribuidos de manera irregular en el territorio. Registrando una elevación media desde 0.18 a $215.37 \mathrm{msnm}$, una distancia al mar de 82.18 a $7,207.80 \mathrm{~m}$ y entre 16.04 a $6,255.44 \mathrm{~m}$ de alejamiento de la zona forestal. Mientras que, en cuanto a la composición del terreno, los parques presentaron una pendiente media de $3.88 \%$, con variaciones entre 0.02 a $16.94 \%$.

Por su parte, tanto el área de $L_{\max }$, como aquella entre el límite de enfriamiento y el área de $500 \mathrm{~m}$, varían en cada una de las temporadas. Registrando valores medios diferentes en cada una de éstas. Asimismo, los descriptores estadísticos de los alrededores son estimados únicamente con el área de los casos positivos, los cuales también varían en cantidad. 


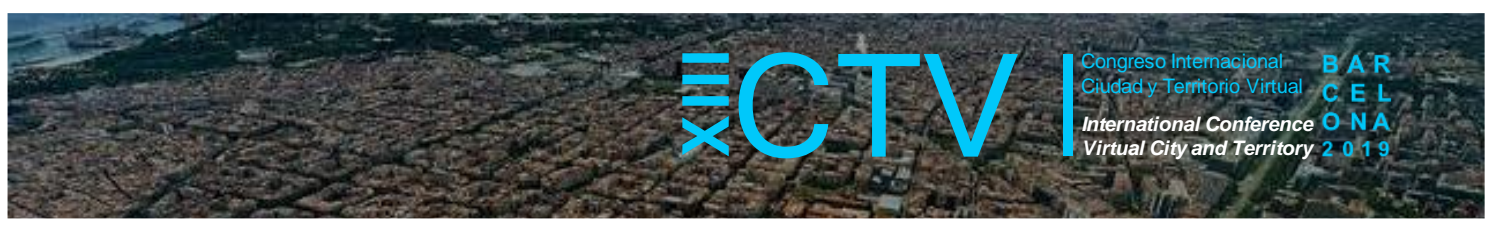

\subsection{Variaciones estacionales en la composición de los espacios verdes}

El NDVI medio de los parques registró poca variación a lo largo del año, oscilando entre los 0.40 en verano como máximo y 0.32 en invierno como mínimo (Tabla 5). La mayor concentración de casos se mantuvo constante en los cuatro periodos con un NDVI medio entre 0.30 y 0.35 . No obstante, en invierno más de la mitad de los parques registraron un NDVI entre 0.20 y 0.35 , mientras que el resto del año se concentraron dentro de 0.30 a $0.45 \mathrm{NDVI}$. Dicha variación de distribución refleja una pérdida importante en la calidad de la vegetación en algunos parques durante el invierno, aunque también se observa su preservación durante todo el año en algunos parques (Figura 7).

Tabla 5. Estadística descriptiva de NDVI de las cuatro temporadas del año

Fuente: Elaboración propia

\begin{tabular}{|l|c|c|c|c|}
\hline \multicolumn{1}{|c}{ NDVI } & $\mathbf{0 1 - 3 1}$ & $\mathbf{0 4 - 2 1}$ & $\mathbf{0 7 - 1 9}$ & $\mathbf{1 0 - 2 3}$ \\
\hline Max & 0.55 & 0.55 & 0.61 & 0.61 \\
\hline Min & 0.09 & 0.15 & 0.13 & 0.10 \\
\hline Mean & $\mathbf{0 . 3 2}$ & $\mathbf{0 . 3 6}$ & $\mathbf{0 . 4 0}$ & $\mathbf{0 . 4 1}$ \\
\hline Std. Dev. & 0.10 & 0.09 & 0.09 & 0.09 \\
\hline
\end{tabular}

Figura 7. Histogramas de los parques por NDVI las cuatro temporadas del año

a) NDVI 2018-01-31

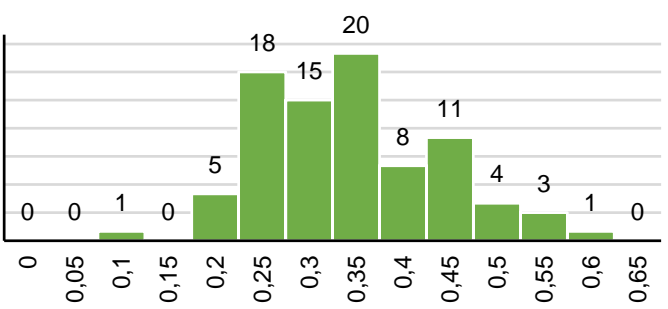

c) NDVI 2018-07-19

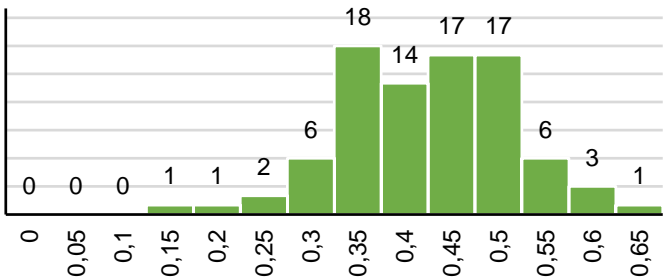

b) NDVI 2018-04-21

21

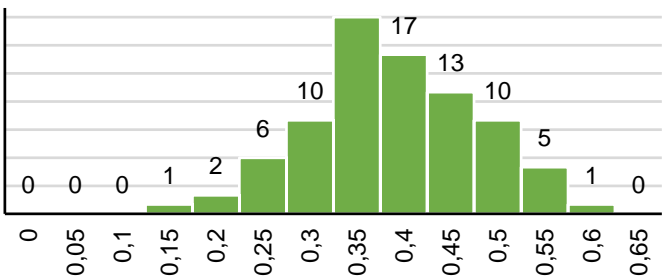

d) NDVI 2018-10-23

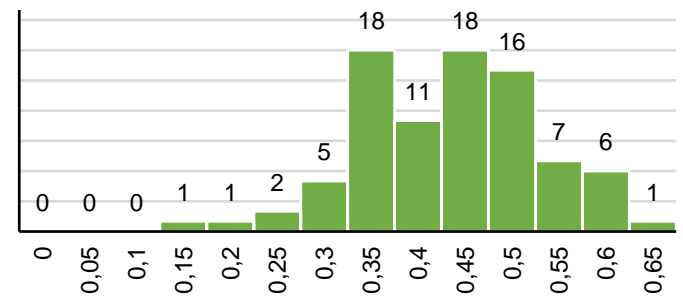

Fuente: Elaboración propia

La variación de la composición de los espacios verdes durante el año se observa con mayor detalle en las áreas divididas por rangos de NDVI (Figura 8). En la superficie total al interior de los parques se observa la predominante presencia de los rangos con vegetación. No obstante, en invierno las superficies con $N D V I<0.20$ y $N D V I<0.30$ alcanzan el 22 y $24 \%$ de la superficie total de los parques respectivamente, siendo la temporada con la menor presencia de vegetación dentro de los parques. Asimismo, las áreas con NDVI $>0.60$ apenas alcanzan el $7 \%$ de la superficie total de los parques durante verano y otoño, así como 3 y $2 \%$ en primavera e invierno. 


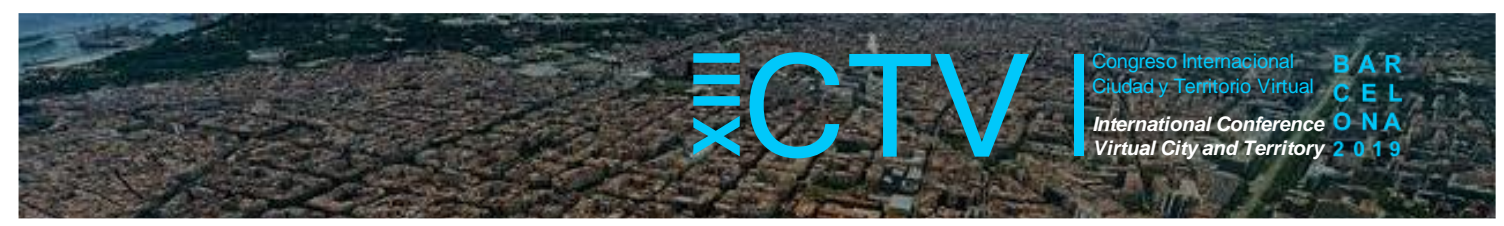

El rango con mayor variación anual fue el de NDVI entre 0.50 a 0.60 con un aumento del $15 \%$ en la cubierta de las cubiertas con vegetación de invierno a otoño de 2018.

Figura 8. Porcentaje de superficie por rangos de NDVI en el conjunto de parques y sus alrededores

a) NDVI parques

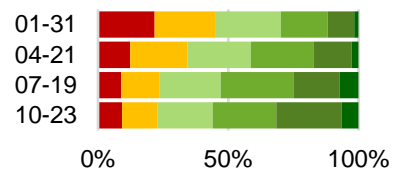

$10-23 \quad 07-1904-21 \quad 01-31$ \begin{tabular}{|c|c|c|c|c|}
\hline$-<0.2$ & $9 \%$ & $9 \%$ & $12 \%$ & $22 \%$ \\
\hline
\end{tabular}

\begin{tabular}{|l|l|l|l|l|}
\hline $0.2-0.3$ & $14 \%$ & $15 \%$ & $22 \%$ & $24 \%$ \\
\hline
\end{tabular}

\begin{tabular}{|l|l|l|l|l|}
\hline$-0.3-0.4$ & $21 \%$ & $24 \%$ & $24 \%$ & $25 \%$ \\
\hline
\end{tabular}

\begin{tabular}{|l|l|l|l|l|}
\hline$-0.4-0.5$ & $24 \%$ & $28 \%$ & $24 \%$ & $18 \%$ \\
\hline
\end{tabular}

\begin{tabular}{|c|c|c|c|c|}
\hline$-0.5-0.6$ & $25 \%$ & $18 \%$ & $15 \%$ & $10 \%$ \\
\hline
\end{tabular}

\begin{tabular}{|l|l|l|l|l|}
\hline$->0.6$ & $7 \%$ & $7 \%$ & $3 \%$ & $2 \%$ \\
\hline
\end{tabular} b) NDVI Área Lmax

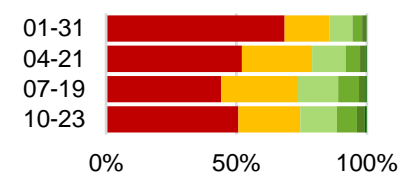

$\begin{array}{llll}10-23 & 07-19 & 04-21 & 01-31\end{array}$ \begin{tabular}{|l|l|l|l|l|}
\hline$-<0.2$ & $51 \%$ & $44 \%$ & $52 \%$ & $69 \%$ \\
\hline
\end{tabular}

\begin{tabular}{|l|l|l|l|l|}
\hline$-0.2-0.3$ & $24 \%$ & $29 \%$ & $27 \%$ & $17 \%$ \\
\hline
\end{tabular}

\begin{tabular}{|l|l|l|l|l|}
\hline $10.3-0.4$ & $14 \%$ & $15 \%$ & $13 \%$ & $9 \%$ \\
\hline
\end{tabular}

\begin{tabular}{|l|l|l|l|l|}
\hline $\mathbf{0} 0.4-0.5$ & $8 \%$ & $8 \%$ & $5 \%$ & $4 \%$ \\
\hline
\end{tabular}

\begin{tabular}{|l|l|l|l|l|}
\hline$-0.5-0.6$ & $3 \%$ & $2 \%$ & $2 \%$ & $2 \%$ \\
\hline
\end{tabular}

\begin{tabular}{|l|l|l|l|l|}
\hline $\mathbf{m}>0.6$ & $1 \%$ & $1 \%$ & $0 \%$ & $0 \%$ \\
\hline
\end{tabular} c) NDVI fuera de Área Lmax

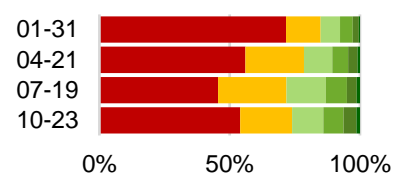

$10-2307-1904-2101-31$ \begin{tabular}{|l|l|l|l|l|}
\hline$-<0.2$ & $54 \%$ & $46 \%$ & $56 \%$ & $72 \%$ \\
\hline
\end{tabular}

\begin{tabular}{|l|l|l|l|l|}
\hline $0.2-0.3$ & $20 \%$ & $26 \%$ & $22 \%$ & $13 \%$ \\
\hline
\end{tabular}

\begin{tabular}{|l|l|l|l|l|}
\hline$-0.3-0.4$ & $12 \%$ & $15 \%$ & $11 \%$ & $7 \%$ \\
\hline
\end{tabular}

\begin{tabular}{|c|c|c|c|c|}
$-0.4-0.5$ & $8 \%$ & $8 \%$ & $6 \%$ & $5 \%$ \\
\hline
\end{tabular}

\begin{tabular}{|l|l|l|l|l|}
\hline$-0.5-0.6$ & $5 \%$ & $4 \%$ & $4 \%$ & $2 \%$ \\
\hline
\end{tabular}

\begin{tabular}{|l|l|l|l|l|}
\hline $\mathbf{n}>0.6$ & $1 \%$ & $1 \%$ & $1 \%$ & $0 \%$ \\
\hline
\end{tabular}

Fuente: Elaboración propia

En cuanto a los alrededores urbanos, se registró una predominante superficie sin vegetación. Primeramente, el área de extensión del efecto de enfriamiento registró más del $50 \%$ de superficies con $N D V K<0.20$ durante tres temporadas del año, aunque verano registró un $44 \%$ de $N D V I<0.20$ y $29 \%$ con $N D V I$ entre 0.20 a 0.30 . Así como, durante verano y otoño se registró apenas el $1 \%$ de superficie con $N D V l>0.60$, mientras que invierno y primavera no se registró. Además, la mayor variación de porcentaje de cubierta de vegetación se registró entre invierno y verano, con un aumento del $24 \%$ de la cubierta con vegetación durante verano. Por otra parte, los alrededores más allá del área de enfriamiento de los parques, registraron un comportamiento similar. Donde predominan las superficies con $N D V K<0.20$, y se reducen un $26 \%$ de la superficie cubierta de invierno a verano.

\subsection{Correlación bivariada entre atributos físicos e indicadores de enfriamiento}

Las correlaciones bivariadas entre los indicadores de enfriamiento y los atributos físicos apuntaron a una predominante influencia de las características propias al interior del parque y no de su entorno (Tabla 4). Particularmente, los atributos físicos relacionados con la composición de los espacios verdes registraron una influencia predominante durante las cuatro temporadas.

Los valores medios de $L S T$ y NDVI de los parques registraron una correlación significativa con los indicadores de enfriamiento durante todo el año, positiva y negativa respectivamente. No obstante, en los alrededores, únicamente las áreas más allá de la extensión de enfriamiento registraron correlación significativa positiva con la $L S T$ durante invierno con los tres indicadores y durante primavera únicamente con la extensión. Esto refiere a que cuanto mayor es la temperatura en los alrededores de un parque, su extensión de enfriamiento tiende a ser mayor. En cuanto a la forma de los parques, se registró influencia significativa durante primavera, verano y otoño. En primavera, el Área del parque registró influencia significativa positiva con la intensidad de enfriamiento, lo que implica que una mayor superficie tiende a generar una mayor intensidad de enfriamiento. Mientras que el LS/ registró una correlación significativa negativa con 


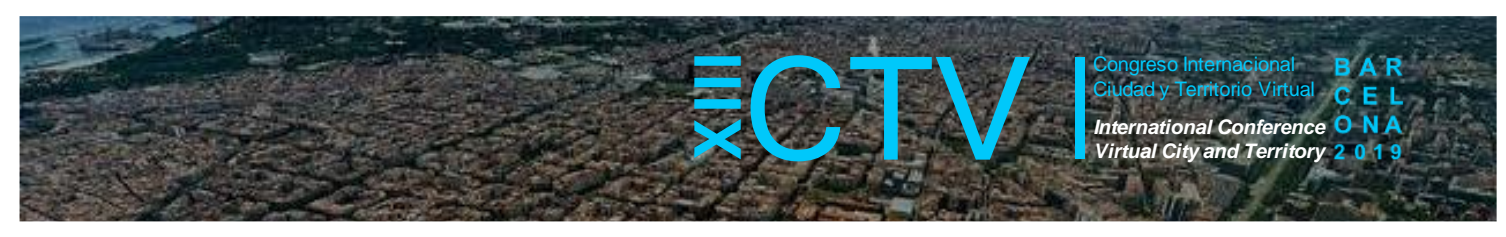

la extensión y la intensidad durante verano y otoño, lo que indica que cuanto más compleja es la forma de un parque, su efecto de enfriamiento tiende a ser menor.

Figura 9. Correlación bivariada entre indicadores de $\mathrm{PCl}$ y los descriptores físicos

\begin{tabular}{|c|c|c|c|c|c|c|c|c|c|c|}
\hline \multirow{2}{*}{\multicolumn{3}{|c|}{$N=86$}} & \multicolumn{2}{|c|}{ 2018-01-31 } & \multicolumn{2}{|c|}{ 2018-04-21 } & \multicolumn{2}{|c|}{ 2018-07-19 } & \multicolumn{2}{|c|}{ 2018-10-23 } \\
\hline & & & Lmax & $\Delta \operatorname{Tmax}$ & Lmax & $\Delta \operatorname{Tmax}$ & Lmax & $\Delta \operatorname{Tmax}$ & Lmax & $\Delta \operatorname{Tmax}$ \\
\hline \multirow{2}{*}{\multicolumn{2}{|c|}{$\overline{\mathrm{PCI}}$}} & Lmax & 1 & $.660^{\star \star}$ & 1 & $.546^{\star \star}$ & 1 & $.460^{\star \star}$ & 1 & $.519^{\star \star}$ \\
\hline & & $\Delta \operatorname{Tmax}$ & $.660^{\star \star}$ & 1 & $.546^{\star \star}$ & 1 & $.460^{\star *}$ & 1 & $.519^{\star \star}$ & 1 \\
\hline \multirow[t]{14}{*}{ Parque } & \multirow[t]{2}{*}{ Forma } & Area & 0.069 & 0.15 & 0.072 & $.260^{*}$ & 0.052 & 0.174 & 0.038 & 0.199 \\
\hline & & LSI & -0.093 & -0.1 & -0.204 & -0.128 & $-.244^{*}$ & $-.324^{\star *}$ & $-.238^{*}$ & $-.301^{\star *}$ \\
\hline & \multirow[t]{2}{*}{ İndices } & LST & $.441^{\star \star}$ & $-.496^{\star *}$ & $-.298^{\star \star}$ & $-.692^{\star *}$ & $-.300^{\star *}$ & $-.683^{* *}$ & $-.360^{\star *}$ & $-.595^{\star \star}$ \\
\hline & & NDVI & $.376^{\star \star}$ & $.567^{\star \star}$ & $.260^{\star}$ & $.608^{\star \star}$ & $.258^{*}$ & $.627^{\star *}$ & $.257^{\star}$ & $.589^{\star *}$ \\
\hline & \multirow[t]{6}{*}{$\begin{array}{l}\text { \% área } \\
\text { NDVI }\end{array}$} & $<0.20$ &.$\overline{-}$ & & & & & & & $-.493^{\star \star}$ \\
\hline & & $0.20-0.30$ & -0.201 & $-.250^{*}$ & -0.075 & $-.369^{\star \star}$ & -0.163 & $-.492^{\star \star}$ & $-.232^{*}$ & $-.371^{\star *}$ \\
\hline & & $0.30-0.40$ & $.213^{\star}$ & 0.083 & 0.132 & $-.225^{\star}$ & -0.038 & $-.276^{\star \star}$ & -0.063 & $-.235^{\star}$ \\
\hline & & $0.40-0.50$ & $.322^{\star \star}$ & $.349^{\star *}$ & $.244^{\star}$ & $.447^{\star \star}$ & $.252^{*}$ & 0.183 & 0.171 & 0.137 \\
\hline & & $0.50-0.60$ & 0.208 & $.483^{\star \star}$ & 0.132 & $.582^{\star \star}$ & 0.118 & $.603^{\star \star}$ & 0.212 & $.485^{\star \star}$ \\
\hline & & $>0.60$ & 0.091 & 0.206 & -0.105 & 0.113 & 0.064 & $.345^{\star *}$ & 0.035 & $.313^{* *}$ \\
\hline & \multirow[t]{4}{*}{ Ubicación } & Elevación & 0.108 & 0.119 & 0.048 & $.254^{*}$ & 0.026 & $.244^{*}$ & 0.022 & 0.151 \\
\hline & & Pendiente & 0.146 & 0.082 & 0.018 & $.342^{\star \star}$ & -0.033 & $.259^{\star}$ & -0.01 & 0.198 \\
\hline & & Dist. mar & 0.107 & 0.057 & 0.136 & 0.129 & 0.124 & 0.178 & 0.06 & 0.091 \\
\hline & & Dist bosque & -0.145 & -0.077 & -0.185 & -0.146 & -0.171 & -0.209 & -0.105 & -0.119 \\
\hline \multirow{12}{*}{$\begin{array}{l}\text { Área } \\
\text { Lmax }\end{array}$} & \multirow[t]{2}{*}{ Índice } & LST & -0.174 & 0.01 & 0.099 & -0.1 & 0.001 & -0.077 & -0.119 & -0.098 \\
\hline & & NDVI & 0.106 & 0 & -0.051 & -0.013 & 0.105 & -0.027 & 0.074 & -0.062 \\
\hline & \multirow{6}{*}{$\begin{array}{l}\text { \% área } \\
\text { NDVI }\end{array}$} & $<0.20$ & -0.134 & -0.034 & 0.075 & 0.072 & -0.059 & 0.099 & -0.015 & 0.12 \\
\hline & & $0.20-0.30$ & -0.04 & -0.124 & -0.083 & -0.147 & -0.09 & -0.195 & $-.226^{*}$ & -0.191 \\
\hline & & $0.30-0.40$ & 0.116 & 0.074 & -0.076 & -0.143 & 0.105 & -0.027 & 0.048 & -0.155 \\
\hline & & $0.40-0.50$ & $.239^{\star}$ & 0.165 & -0.001 & 0.117 & 0.102 & 0.01 & 0.116 & 0.042 \\
\hline & & $0.50-0.60$ & 0.195 & 0.075 & 0.003 & 0.164 & 0.093 & 0.059 & 0.179 & 0.034 \\
\hline & & $>0.60$ & $.217^{\star}$ & -0.015 & -0.017 & 0.047 & 0.045 & -0.057 & 0.075 & -0.04 \\
\hline & \multirow[t]{4}{*}{ Ubicación } & Elevación & 0.109 & 0.109 & 0.028 & 0.201 & 0.011 & 0.208 & 0.044 & 0.12 \\
\hline & & Pendiente & 0.139 & 0.088 & -0.006 & $.320^{\star *}$ & -0.036 & $.246^{\star}$ & 0.036 & 0.197 \\
\hline & & Dist. mar & 0.107 & 0.053 & 0.122 & 0.112 & 0.11 & 0.165 & 0.164 & 0.162 \\
\hline & & Dist bosque & -0.142 & -0.07 & -0.167 & -0.125 & -0.151 & -0.192 & -0.19 & -0.16 \\
\hline \multirow{12}{*}{$\begin{array}{l}\text { Fuera } \\
\text { de } \\
\text { Área } \\
\text { Lmax }\end{array}$} & \multirow[t]{2}{*}{ Índice } & LST & $.236^{\star}$ & $.356^{\star \star}$ & $.240^{*}$ & 0.068 & 0.189 & 0.159 & 0.065 & 0.096 \\
\hline & & NDVI & 0.12 & 0.082 & -0.072 & 0.041 & -0.018 & -0.006 & -0.037 & -0.06 \\
\hline & \multirow{6}{*}{$\begin{array}{l}\text { \% área } \\
\text { NDVI }\end{array}$} & $<0.20$ & -0.113 & -0.074 & 0.08 & 0.007 & 0.008 & 0.01 & 0.057 & 0.096 \\
\hline & & $0.20-0.30$ & 0.105 & 0.035 & 0.044 & -0.119 & 0.206 & 0.106 & -0.004 & -0.076 \\
\hline & & $0.30-0.40$ & 0.139 & 0.068 & -0.103 & -0.026 & -0.038 & -0.016 & -0.04 & -0.123 \\
\hline & & $0.40-0.50$ & 0.093 & 0.068 & -0.108 & 0.05 & -0.06 & -0.061 & -0.075 & -0.103 \\
\hline & & $0.50-0.60$ & 0.057 & 0.112 & -0.117 & 0.103 & -0.112 & -0.071 & -0.106 & -0.065 \\
\hline & & $>0.60$ & 0.103 & 0.16 & -0.131 & 0.065 & -0.191 & -0.041 & 0.018 & 0.037 \\
\hline & \multirow[t]{4}{*}{ Ubicación } & Elevación & 0.108 & 0.104 & 0.045 & 0.165 & 0.036 & 0.196 & 0.048 & 0.115 \\
\hline & & Pendiente & 0.067 & 0.032 & -0.005 & 0.212 & -0.011 & 0.183 & -0.032 & 0.099 \\
\hline & & Dist. mar & 0.103 & 0.051 & 0.122 & 0.107 & 0.112 & 0.16 & 0.081 & 0.105 \\
\hline & & Dist bosque & -0.136 & -0.067 & -0.168 & -0.122 & -0.154 & -0.188 & -0.117 & -0.127 \\
\hline
\end{tabular}

Fuente: Elaboración propia

Los descriptores de ubicación de los parques y sus alrededores registraron influencia significativa positiva con la intensidad y gradiente durante primavera y verano. Lo que indica que, una mayor pendiente en el terreno y más elevación sobre el nivel del mar, tanto del parque y sus 


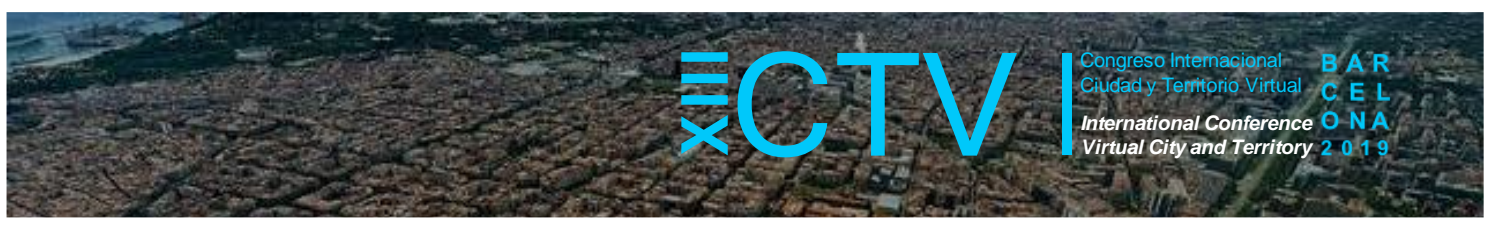

alrededores, tienden a derivar en una mayor intensidad y gradiente. Aunque esto se condiciona a las temporadas con las temperaturas más elevadas del año.

\subsection{Modelo explicativo de $\Delta T_{\max }$}

Los resultados de las regresiones múltiples apuntan las características físicas de los parques y sus alrededores que influyen sobre su efecto de enfriamiento son distintas en cada una de las temporadas. Donde la intensidad de enfriamiento registró los valores de correlación múltiple más elevados (Figura 10).

Figura 10. Resultado de regresión múltiple entre $\Delta T_{\max }$ y variables físicas

a) $\Delta T \max 01-31$

\begin{tabular}{|l|c|c|c|}
\hline \multicolumn{1}{|c|}{$\mathbf{R}$} & \multicolumn{1}{|c|}{$\mathbf{R}^{\mathbf{2}}$} & $\begin{array}{c}\mathbf{R}^{\mathbf{2}} \\
\text { ajustada }\end{array}$ & $\begin{array}{c}\text { Error } \\
\text { estándar }\end{array}$ \\
\hline 0.791 & 0.626 & 0.597 & 0.261 \\
\hline \multicolumn{1}{|c|}{ Modelo } & $\begin{array}{c}\text { Coefs. no } \\
\text { estandarizados }\end{array}$ & Sig. & VIF \\
\hline (Constante) & 1.301 & 0 & \\
\hline $\begin{array}{l}\text { Parque } \\
\text { NDVI < 0.20 }\end{array}$ & -1.402 & 0 & 2.133 \\
\hline $\begin{array}{l}\text { Parque } \\
\text { NDVI 0.40 - 0.50 }\end{array}$ & 0.605 & 0.012 & 1.876 \\
\hline $\begin{array}{l}\text { Parque } \\
\text { NDVI 0.50 - 0.60 }\end{array}$ & $\mathbf{1 . 2 2 1}$ & $\mathbf{0}$ & $\mathbf{1 . 7 4 3}$ \\
\hline $\begin{array}{l}\text { Área Lmax } \\
\text { NDVI 0.20 - 0.30 }\end{array}$ & -1.119 & 0.003 & 1.46 \\
\hline $\begin{array}{l}\text { Área Lmax } \\
\text { NDVI 0.30 - 0.40 }\end{array}$ & -1.038 & 0.01 & 2.001 \\
\hline $\begin{array}{l}\text { Fuera Lmax } \\
\text { Pendiente }\end{array}$ & -0.058 & 0 & 2.084 \\
\hline
\end{tabular}

c) $\Delta T \max 07-19$

\begin{tabular}{|c|c|c|c|}
\hline $\mathbf{R}$ & $\mathbf{R}^{2}$ & $\begin{array}{c}\mathbf{R}^{2} \\
\text { ajustada }\end{array}$ & $\begin{array}{c}\text { Error } \\
\text { estándar }\end{array}$ \\
\hline 0.772 & 0.597 & 0.565 & 0.56 \\
\hline
\end{tabular}

\begin{tabular}{|l|c|c|c|}
\hline \multicolumn{1}{|c|}{ Modelo } & $\begin{array}{c}\text { Coefs. no } \\
\text { estandarizados }\end{array}$ & Sig. & VIF \\
\hline (Constante) & 1.212 & 0.001 & \\
\hline $\begin{array}{l}\text { Parque } \\
\text { LSI }\end{array}$ & -0.35 & 0.008 & 1.109 \\
\hline $\begin{array}{l}\text { Parque } \\
\text { Slope }\end{array}$ & 0.118 & 0.001 & 5.512 \\
\hline $\begin{array}{l}\text { Parque } \\
\text { NDVI 0.20 - 0.30 }\end{array}$ & -1.313 & 0.033 & 1.62 \\
\hline $\begin{array}{l}\text { Parque } \\
\text { NDVI 0.50 - 0.60 }\end{array}$ & $\mathbf{2 . 9 1 7}$ & $\mathbf{0}$ & $\mathbf{1 . 6 3 1}$ \\
\hline $\begin{array}{l}\text { Fuera Lmax } \\
\text { NDVl<0.20 }\end{array}$ & 1.612 & 0 & 1.426 \\
\hline $\begin{array}{l}\text { Fuera Lmax } \\
\text { Pendiente }\end{array}$ & -0.124 & 0.023 & 6.14 \\
\hline
\end{tabular}

b) $\Delta \operatorname{Tmax} 04-21$

\begin{tabular}{|l|c|c|c|}
\multicolumn{1}{|c|}{$\mathbf{R}$} & \multicolumn{1}{|c|}{$\mathbf{R}^{\mathbf{2}}$} & $\begin{array}{c}\mathbf{R}^{2} \\
\text { ajustada }\end{array}$ & $\begin{array}{c}\text { Error } \\
\text { estándar }\end{array}$ \\
\hline \multicolumn{1}{|c|}{0.770} & 0.592 & 0.571 & 0.561 \\
\hline \multicolumn{1}{|c|}{ Modelo } & $\begin{array}{c}\text { Coefs. no } \\
\text { estandarizados }\end{array}$ & Sig. & VIF \\
\hline (Constante) & -0.293 & 0.214 & \\
\hline $\begin{array}{l}\text { Parque } \\
\text { Área }\end{array}$ & 0.005 & 0.016 & 1.028 \\
\hline $\begin{array}{l}\text { Parque } \\
\text { NDVI 0.40 - 0.50 }\end{array}$ & 2.138 & 0 & 1.09 \\
\hline $\begin{array}{l}\text { Parque } \\
\text { NDVI 0.50 - 0.60 }\end{array}$ & $\mathbf{2 . 9 4 1}$ & $\mathbf{0}$ & $\mathbf{1 . 2 2 2}$ \\
\hline $\begin{array}{l}\text { Fuera Lmax } \\
\text { NDVI < 0.20 }\end{array}$ & 1.606 & 0 & 1.233 \\
\hline
\end{tabular}

d) $\Delta \operatorname{Tmax} 10-23$

\begin{tabular}{|c|c|c|c|}
\hline $\mathbf{R}$ & $\mathbf{R}^{2}$ & $\begin{array}{c}\mathbf{R}^{2} \\
\text { ajustada }\end{array}$ & $\begin{array}{c}\text { Error } \\
\text { estándar }\end{array}$ \\
\hline 0.728 & 0.531 & 0.494 & 0.401 \\
\hline
\end{tabular}

\begin{tabular}{|l|c|c|c|}
\hline \multicolumn{1}{|c|}{ Modelo } & $\begin{array}{c}\text { Coefs. no } \\
\text { estandarizados }\end{array}$ & Sig. & VIF \\
\hline (Constante) & 0.688 & 0.002 & \\
\hline $\begin{array}{l}\text { Parque } \\
\text { Área }\end{array}$ & 0.004 & 0.006 & 1.017 \\
\hline $\begin{array}{l}\text { Parque } \\
\text { NDVI < 0.20 }\end{array}$ & -2.162 & 0 & 1.645 \\
\hline $\begin{array}{l}\text { Parque } \\
\text { NDVI 0.30 - 0.40 }\end{array}$ & -0.855 & 0.048 & 1.912 \\
\hline $\begin{array}{l}\text { Parque } \\
\text { NDVI 0.50 - 0.60 }\end{array}$ & $\mathbf{0 . 6 4 1}$ & $\mathbf{0 . 0 5 1}$ & $\mathbf{2 . 1 2 2}$ \\
\hline $\begin{array}{l}\text { Parque } \\
\text { NDVI > 0.60 }\end{array}$ & 1.006 & 0.026 & 1.464 \\
\hline $\begin{array}{l}\text { Fuera Lmax } \\
\text { NDVI < } \mathbf{0 . 2 0}\end{array}$ & 1.453 & 0 & 1.419 \\
\hline
\end{tabular}

Fuente: Elaboración propia

Durante invierno, se registró el modelo de la $\Delta T_{\max }$ con la $R^{2}$ ajustada más elevada de las cuatro temporadas con 0.597. En la cual se identificó que, al interior del parque, cuanto mayor es la superficie con $N D V k 0.20$ la $\Delta T_{\max }$ disminuye, mientras que cuanto mayor es la superficie con NDVI entre 0.40 a 0.60 ésta aumenta. Por otra parte, se identificó que cuanto mayor es la 


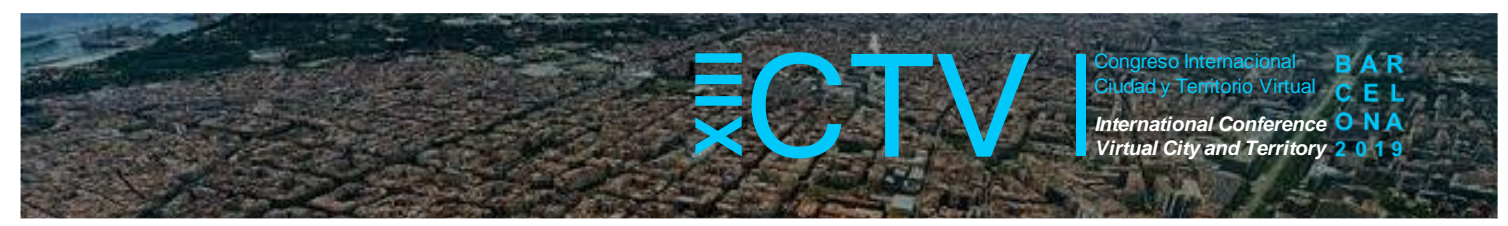

superficie con $N D V I$ entre 0.20 a 0.40 en los alrededores dentro del área de $L_{\max }$, menor es la intensidad. Así como cuanto mayor es la pendiente más allá del límite de enfriamiento menor es la $\Delta T_{\max }$. Este comportamiento apunta que cuanto mayor vegetación al interior del parque, la $\Delta T_{\max }$ incrementa, aunque esta se verá disminuida si el aumento de vegetación se extiende hacia los alrededores urbanos inmediatos. Particularmente, las principales protagonistas durante invierno son las superficies artificiales al interior de los parques, las cuales por cada aumento de $10 \%$ generarían una reducción de $0.14^{\circ} \mathrm{C}$ de $\Delta T_{\max }$.

En primavera, se registró que cuanto mayor es el área, las superficies con NDVI 0.40 a 0.60 al interior del parque, y aquellas con $N D V I<0.20$ en los alrededores inmediatos, mayor será la $\Delta T_{\max }$. Especialmente, se registró la mayor influencia por parte de las superficies con NDVI 0.50 a 0.60 , donde un aumento del $10 \%$ de éstas, derivaría en un aumento de $0.294^{\circ} \mathrm{C}$ de $\Delta T_{\max }$.

En cuanto a verano, los resultados mostraron que el LSI de los parques influye negativamente sobre la $\Delta T_{\max }$. Así como lo hacen las superficies con $N D V I 0.20$ a 0.30 al interior del parque, y la pendiente más allá del límite de enfriamiento. Mientras que la pendiente al interior del parque, las superficies con NDVI 0.50 a 0.60 al interior del parque y aquellas con $N D V I<0.20$ en los alrededores dentro del área de extensión, influyen positivamente. Particularmente, los valores obtenidos apuntan a que el aumento de las superficies con NDVI menor a 0.30 en el parque, disminuirían la $\Delta T_{\max }$, mientras que aumentar aquellas sin vegetación en los alrededores inmediatos la aumentarían.

En cuanto al atributo más influyente, al igual que en primavera corresponde a las superficies con NDVI 0.50 a 0.60 dentro del parque, aumentando la $\Delta T_{\max } 0.291^{\circ} \mathrm{C}$ por cada $10 \%$ de aumento. Por su parte, en otoño se registra el área del parque como atributo significativo. Así como se integran por primera vez las superficies con $N D V l>0.60$ al interior del parque con influencia positiva. Aquí, al igual que en invierno, la mayor influencia la registraron las superficies con NDVI $<0.20$ al interior del parque. Donde un aumento de $10 \%$ de éstas generaría una reducción de $0.216^{\circ} \mathrm{C}$ de $\Delta T_{\max }$.

En general, el único descriptor que registró significancia constante durante las cuatro temporadas es el de las superficies con NDVI 0.50 a 0.60 al interior del parque. Las cuales influyen positivamente sobre el efecto de enfriamiento durante todo el año, oscilando entre los $0.0641^{\circ} \mathrm{C}$ de primavera y $0.294^{\circ} \mathrm{C}$ de otoño por cada $10 \%$ de aumento de dichas superficies.

\subsection{Modelo explicativo de $L_{\max }$}

La regresión lineal múltiple con la $L_{\max }$ como variable dependiente, registró a la $\Delta T_{\max }$ como descriptor constante durante las cuatro temporadas del año (Figura 11). Donde el aumento de $1^{\circ} \mathrm{C}$ de $\Delta T_{\max }$ aumentaría la $L_{\max }$ en $41.82 \mathrm{~m}$ en invierno, $20.22 \mathrm{~m}$ en primavera, $36.29 \mathrm{~m}$ en verano y $23.97 \mathrm{~m}$ en otoño. Especialmente, se registró que en primavera y otoño es la única característica con influencia significativa sobre la extensión de enfriamiento. Donde en ambos casos se obtuvo la $R^{2}$ más baja del año con 0.235 y 0.206 respectivamente. 


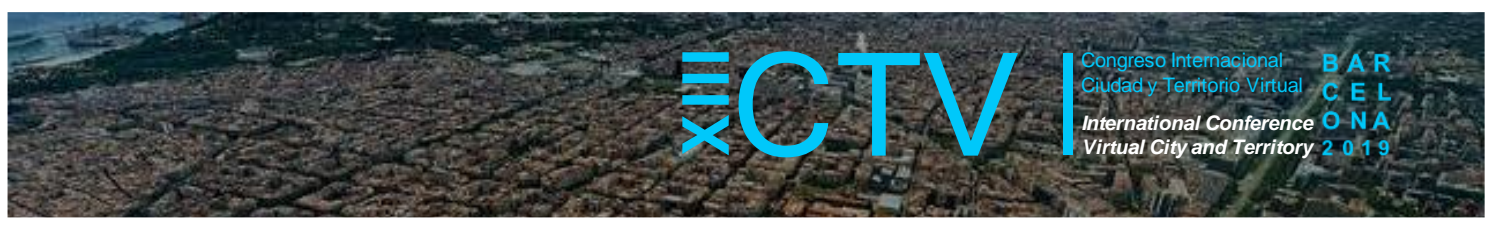

Figura 11. Resultado de regresión múltiple entre $L_{\max }$ y variables físicas

a) Lmax 01-31

\begin{tabular}{|l|c|c|c|}
\multicolumn{1}{|c|}{$\mathbf{R}$} & \multicolumn{1}{|c|}{$\mathbf{R}^{\mathbf{2}}$} & $\begin{array}{c}\mathbf{R}^{\mathbf{2}} \\
\text { ajustada }\end{array}$ & $\begin{array}{c}\text { Error } \\
\text { estándar }\end{array}$ \\
\hline \multicolumn{1}{|c|}{0.746} & 0.556 & 0.527 & 15.604 \\
\hline \multicolumn{1}{|c|}{ Modelo } & $\begin{array}{c}\text { Coefs. no } \\
\text { estandarizados }\end{array}$ & Sig. & VIF \\
\hline (Constante) & 29.001 & .000 & \\
\hline $\begin{array}{l}\text { Parque } \\
\text { NDVI 0.50 - 0.60 }\end{array}$ & -53.620 & .001 & 2.129 \\
\hline $\begin{array}{l}\text { Área Lmax } \\
\text { NDVI 0.40 - 0.50 }\end{array}$ & 183.609 & .001 & 2.585 \\
\hline $\begin{array}{l}\text { Área Lmax } \\
\text { NDVI > 0.60 }\end{array}$ & 859.885 & .001 & 1.143 \\
\hline $\begin{array}{l}\text { Fuera Lmax } \\
\text { NDVI 0.50 - 0.60 }\end{array}$ & -164.300 & .040 & 2.460 \\
\hline \begin{tabular}{l}
$\mathbf{\Delta T m a x}$ \\
\hline
\end{tabular} & $\mathbf{4 1 . 8 2 0}$ & .000 & $\mathbf{1 . 3 6 4}$ \\
\hline
\end{tabular}

c) Lmax 07-19

\begin{tabular}{|c|c|c|c|}
\hline $\mathbf{R}$ & $\mathbf{R}^{2}$ & $\begin{array}{c}\mathbf{R}^{2} \\
\text { ajustada }\end{array}$ & $\begin{array}{c}\text { Error } \\
\text { estándar }\end{array}$ \\
\hline 0.627 & 0.393 & 0.345 & 30.996 \\
\hline
\end{tabular}

\begin{tabular}{|l|c|c|c|}
\hline \multicolumn{1}{|c|}{ Modelo } & $\begin{array}{c}\text { Coefs. no } \\
\text { estandarizados }\end{array}$ & Sig. & VIF \\
\hline (Constant) & 92.879 & .000 & \\
\hline $\begin{array}{l}\text { Parque } \\
\text { Pendiente }\end{array}$ & -6.634 & .001 & 5.924 \\
\hline $\begin{array}{l}\text { Parque } \\
\text { NDVI 0.50 - 0.60 }\end{array}$ & -124.207 & .000 & 2.232 \\
\hline $\begin{array}{l}\text { Área Lmax } \\
\text { NDVI < 0.20 }\end{array}$ & -94.437 & .000 & 1.901 \\
\hline $\begin{array}{l}\text { Fuera Lmax } \\
\text { Pendiente }\end{array}$ & 6.818 & .026 & 6.262 \\
\hline $\begin{array}{l}\text { Fuera Lmax } \\
\text { NDVI > 0.60 }\end{array}$ & -691.303 & .016 & 1.439 \\
\hline $\mathbf{\Delta T m a x}$ & $\mathbf{3 6 . 2 9 2}$ & .000 & $\mathbf{2 . 1 1 5}$ \\
\hline
\end{tabular}

Fuente: Elaboración propia b) Lmax 04-21

\begin{tabular}{|c|c|c|c|}
\hline $\mathbf{R}$ & $\mathbf{R}^{2}$ & $\begin{array}{c}\mathbf{R}^{2} \\
\text { ajustada }\end{array}$ & $\begin{array}{c}\text { Error } \\
\text { estándar }\end{array}$ \\
\hline 0.485 & 0.235 & 0.226 & 31.433 \\
\hline
\end{tabular}

\begin{tabular}{|l|c|c|c|}
\hline \multicolumn{1}{|c|}{ Modelo } & $\begin{array}{c}\text { Coefs. no } \\
\text { estandarizados }\end{array}$ & Sig. & VIF \\
\hline (Constant) & 43.518 & .000 & \\
\hline$\Delta$ Tmax & 20.217 & .000 & 1.000 \\
\hline
\end{tabular}

d) $\operatorname{Lmax} 10-23$

\begin{tabular}{|c|c|c|c|}
\hline $\mathbf{R}$ & $\mathbf{R}^{2}$ & $\begin{array}{c}\mathbf{R}^{2} \\
\text { ajustada }\end{array}$ & $\begin{array}{c}\text { Error } \\
\text { estándar }\end{array}$ \\
\hline 0.454 & 0.206 & 0.196 & 26.692 \\
\hline
\end{tabular}

\begin{tabular}{|l|c|c|c|}
\hline \multicolumn{1}{|c|}{ Modelo } & $\begin{array}{c}\text { Coefs. no } \\
\text { estandarizados }\end{array}$ & Sig. & VIF \\
\hline (Constant) & 40.943 & .000 & \\
\hline$\Delta$ Tmax & 23.967 & .000 & 1.000 \\
\hline
\end{tabular}

En cuanto a verano e invierno, se observa una mayor influencia de las características físicas de los alrededores urbanos sobre la extensión de enfriamiento de lo que hubo sobre la intensidad. De esta manera, se observa en ambos casos que el aumento de superficie con NDVI 0.50 a 0.60 al interior del parque, generarían una disminución de la extensión de enfriamiento. Particularmente durante invierno, se registró que un aumento de $10 \%$ de las superficies con NDVI 0.40 a 0.50 y $N D V I>0.60$ en los alrededores urbanos dentro del área de extensión, influyen positivamente en la propagación de enfriamiento por 18.36 y $85.99 \mathrm{~m}$ respectivamente. Mientras que, en verano, únicamente la pendiente más allá del límite de enfriamiento influye favorablemente, además de la intensidad.

Los valores en relación a la extensión, señalan que una mayor presencia de vegetación densa en los alrededores inmediatos de los parques, serían favorables para la propagación de enfriamiento. No obstante, también apuntan a que dicha vegetación más allá de los límites de enfriamiento sería desfavorecedor. Estas afirmaciones, se relacionan con lo referido en la correlación bivariada, donde un aumento de temperatura en las zonas urbanas más lejanas a los parques, podrían implicar un mayor efecto de enfriamiento. Dicha condición podría estar 


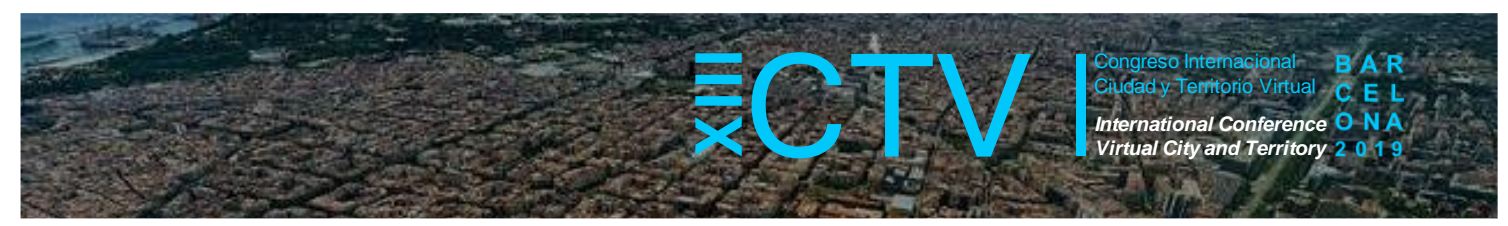

relacionada a la claridad de la definición del efecto de enfriamiento, ya que como se menciona en el punto 3.2, cuanto mayor es la temperatura, el efecto de enfriamiento tiende a verse con mayor claridad sobre el territorio y, por lo tanto, sus indicadores presentan valores más elevados.

\section{Conclusiones}

Los resultados permiten una cuantificación generalizada de la influencia de los atributos físicos de los parques y su entorno urbano sobre su efecto de enfriamiento en las diferentes temporadas del año en una escala local. Primeramente, se identifica que las variaciones de temperatura tanto estacionales como las territoriales derivadas por la complejidad de la estructura urbana y el efecto de isla de calor, influyen determinantemente sobre el efecto de enfriamiento de los parques. Donde una mayor temperatura de superficie y aire, permite identificar con mayor claridad el efecto climático de los parques sobre su entorno urbano mediante el método de homogenización de temperaturas del entorno mediante anillos concéntricos.

Por otra parte, la variación temporal en la composición de los espacios verdes podría representar una fuente de investigaciones venideras en relación al estudio de casos específicos o subclasificaciones espaciales del territorio en relación a las zonas climáticas urbanas en que se encuentran los parques. Esto podría permitir ponderar el peso de la temperatura del ambiente sobre las características físicas propias del parque y su entorno urbano. Para que, con mayor detalle, se puede evaluar el papel que los elementos del medio físico construido juegan sobre las variaciones espacio temporales del efecto de enfriamiento de los parques urbanos.

No obstante, desde la misma perspectiva que se plantea el presente trabajo, se reconoce que la inclusión de un mayor número de casos con contextos climáticos diferentes, podría permitir la inclusión de variables climáticas territoriales en los modelos explicativos que también respondan al reconocimiento del papel de las variaciones meteorológicas.

Finalmente, el presente trabajo registró coincidencias con la literatura existente en relación a la influencia de las características físicas de los parques y su entorno urbano sobre su efecto de enfriamiento. Particularmente, se destacan los valores que competen al contexto particular de Barcelona, los cuales permiten de manera general la proposición de acciones concretas en relación al diseño de los espacios urbanos para la mejora climática de los exteriores. De tal forma que se atiende de manera precisa, la necesidad inminente de reducción del calentamiento de la ciudad ante los siniestros previstos a intensificarse durante episodios extremos de calor. Pero también, asegurando condiciones climáticas adecuadas durante el resto de los días del año, buscando satisfacer las necesidades de los habitantes de Barcelona mediante la intervención de los parques urbanos.

Agradecimientos: El presente estudio es parte del proyecto "Urban-CLIMPLAN. La isla de calor urbana: efectos en el cambio climático y modelado para estrategias de planeamiento territorial y urbano. Aplicación a la región metropolitana de Barcelona" (Ref. BIA2015-68623-R). Financiado por el Ministerio de Economía y Competitividad de España (MINECO) y el Fondo Europeo para el Desarrollo Regional (FEDER).

Conflicto de Intereses: Los autores declaran que no hay conflicto de intereses. 


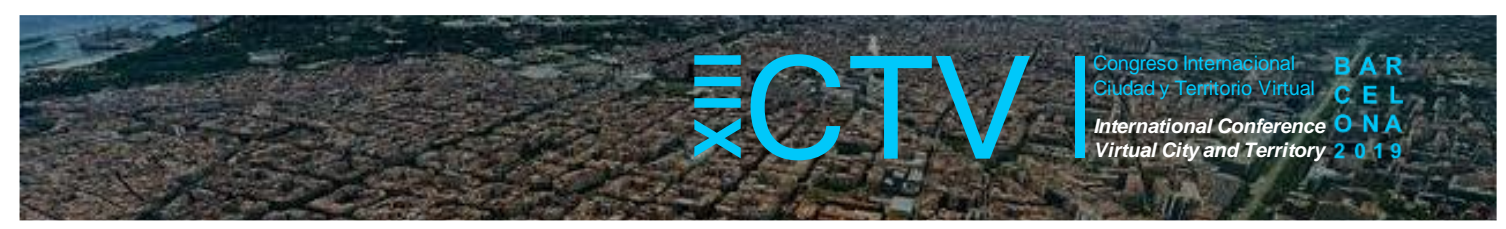

\section{Bibliografía}

Area d'Ecologia i Serveis Urbans de Barcelona. (2017). Límits dels parcs de Barcelona. Direcció Serveis d'Inversions i Espais Vials. Ecologia urbana, Avaluació i Projectes d'Espais Verds. Barcelona: Ajuntament de Barcelona.

Arellano, B., García-Haro, A., \& Roca, J. (2019). Multi-scale approach to quantify the influence of urban green spaces on climate behavior of the Viladecans-Gavà-Castelldefels conurbation in the metropolitan area of Barcelona. European Geosciences Union General Assembly 2019. Vienna. doi:https://www.doi.org/10.1002/essoar.10500946.2

Bowler, D., Buyung-Ali, L., Knight, T., \& Pullin, A. (2010). Urban greening to cool towns and cities: A systematic review of the empirical evidence. Landscape and Urban Planning, 147-155. doi:https://doi.org/10.1016/j.landurbplan.2010.05.006

Cao, X., Onishi, A., Chen, J., \& Imura, H. (2010). Quantifying the cool island intensity of urban parks using ASTER and IKONOS data. Landscape and Urban Planning, 96, 224-231. doi:https://doi.org/10.1016/j.landurbplan.2010.03.008

Departament de Territori i Sostenibilitat. (2016). Mapa urbanístic de Catalunya (MUC). Recuperado el 25 de Julio de 2017, de Generalitat de Catalunya. Departament de Territori i Sostenibilitat:

http://territori.gencat.cat/es/06_territori_i_urbanisme/urbanisme/mapa_urbanistic_de_catalunya/

Hamada, S., \& Ohta, T. (2010). Seasonal variations in the cooling effect of urban green areas on surrounding urban areas. Urban Forestry \& Urban Greening, 9(1), 15-24. doi:https://doi.org/10.1016/j.ufug.2009.10.002

Hamada, S., Tanaka, T., \& Ohta, T. (2013). Impacts of land use and topography on the cooling effect of green areas on surrounding urban areas. Urban Forestry \& Urban Greening, 426-434. doi:https://doi.org/10.1016/j.ufug.2013.06.008

Institut Cartogràfic i Geològic de Catalunya (ICGC). (2017, May 24). WMS OrtoXpres. Recuperado el 17 de Enero de 2019, de ICGC WMS Ortoimatges : https://www.icgc.cat/Administracio-i-empresa/Serveis/Geoinformacio-en-linia-Geoserveis/WMSOrtoimatges/WMS-ortoXpres

Lee, D., Oh, K., \& Seo, J. (2016). An Analysis of Urban Cooling Island (UCI) Effects by Water Spaces Applying UCI Indices. International Journal of Environmental Science and Development, 7(11), 810-815. doi:http://doi.org/10.18178/ijesd.2016.7.11.886

McGarigal, \& Marks, B. J. (1995). FRAGSTATS: spatial pattern analysis program for quantifying landscape structure. General Technical Report, PNW-GTR-351. doi:https://doi.org/10.2737/PNW-GTR-351

Oke, T. (1982). The energetic basis of the urban heat island. Quarterly Journal of the Royal Meteorological Society(108), 1-24. doi:http://dx.doi.org/10.1002/qj.49710845502

Roca, J., Arellano, B., \& Batlle, E. (2018). Green areas and urban heat island: combining remote sensed data with ground observations. Remote Sensing and Modeling of Ecosystems for Sustainability XV, SPIE Proceedings 10767. San Diego. doi:https://doi.org/10.1117/12.2320999 


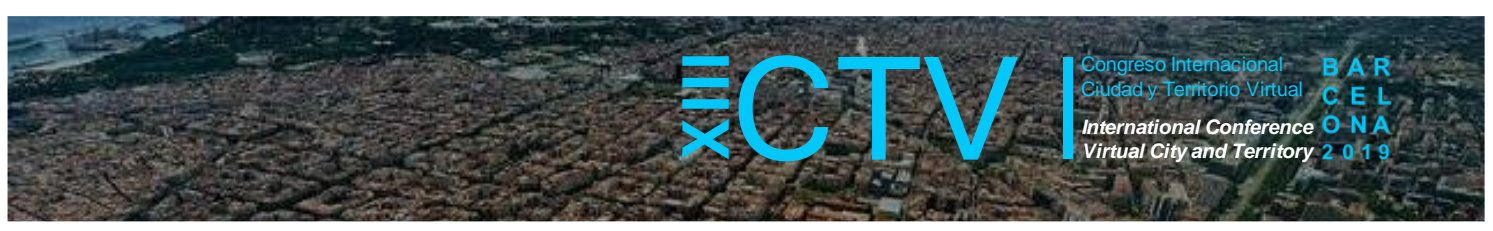

Servei Meteorològic de Catalunya. (2018). Observacions. Estacions automàtiques. Dades d'estacións del Municipi de Barcelona. Recuperado el 24 de Julio de 2017, de Meteo.cat: https://www.meteo.cat/observacions/xema

Sobrino, J., Jiménez-Muñoz, J., Soria, G., Romaguera, M., \& Guanter, L. (2008). Land Surface Emissivity Retrieval From Different VNIR and TIR Sensors. IEEE Transactions on Geoscience and Remote Sensing, 46, 316-327. doi:https://doi.org/10.1109/TGRS.2007.904834

Spronken-Smith, R., \& Oke, T. (1998). The thermal regime of urban parks in two cities with different summer climates. International Journal of Remote Sensing, XIX(11), 2085-2104. doi:http://dx.doi.org/10.1080/014311698214884

Stathopoulou, M., \& Cartalis, C. (2007). Daytime urban heat islands from Landsat ETM+ and Corine land cover data: An application to major cities in Greece. Solar Energy, 358-368. doi:https//doi.org/10.1016/j.solener.2006.06.014

Stathopoulou, M., Cartalis, C., \& Petrakis, M. (2007). Integrating Corine Land Cover data and Landsat TM for surface emissivity definition: application to the urban area of Athens, Greece. International Journal of Remote Sensing, 28(15), 3291-3304. doi:https://doi.org/10.1080/01431160600993421

Statistical Office of the European Union. (2018, Febrero 07). Database. Populations and social conditions. Retrieved from Eurostat: http://ec.europa.eu/eurostat/data/database

U.S. Geological Survey. (2018). Barcelona, Spain. 2018. Obtenido de Landsat-8 OLI/TIRS United States Geological Survey: http://earthexplorer.usgs.gov/ 